\title{
Excitatory-Inhibitory Synaptic Coupling in Avian Nucleus Magnocellularis
}

\author{
Mohammed Al-Yaari, Rei Yamada, and Hiroshi Kuba \\ Department of Cell Physiology, Nagoya University, Graduate School of Medicine, Nagoya 466-8550, Japan
}

The activity of neurons is determined by the balance between their excitatory and inhibitory synaptic inputs. Neurons in the avian nucleus magnocellularis (NM) integrate monosynaptic excitatory and polysynaptic inhibitory inputs from the auditory nerve, and transmit phase-locked output to higher auditory centers. The excitatory input is graded tonotopically, such that neurons tuned to higher frequency receive fewer, but larger, axon terminals. However, it remains unknown how the balance between excitatory and inhibitory inputs is determined in NM. We here examined synaptic and spike responses of NM neurons during stimulation of the auditory nerve in thick brain slices of chicken of both sexes, and found that the excitatory-inhibitory balance varied according to tonotopic region, ensuring reliable spike output across frequencies. Auditory nerve stimulation elicited IPSCs in NM neurons regardless of tonotopic region, but the dependence of IPSCs on intensity varied in a systematic way. In neurons tuned to low frequency, IPSCs appeared and increased in parallel with EPSCs with elevation of intensity, which expanded dynamic range by preventing saturation of spike generation. On the other hand, in neurons tuned to higher frequency, IPSCs were smaller than EPSCs and had higher thresholds for activation, thus facilitating highfidelity transmission. Computer simulation confirmed that these differences in inhibitory input were optimally matched to the patterns of excitatory input, and enabled appropriate level of neuronal output for wide intensity and frequency ranges of sound in the auditory system.

Key words: auditory; brainstem; cochlear nucleus; GABA; inhibition; tonotopy

\section{Significance Statement}

Neurons in nucleus magnocellularis encode timing information of sound across wide intensity ranges by integrating excitatory and inhibitory synaptic inputs from the auditory nerve, but underlying synaptic mechanisms of this integration are not fully understood. We here show that the excitatory-inhibitory relationship was expressed differentially at each tonotopic region; the relationship was linear in neurons tuned to low-frequency, expanding dynamic range by preventing saturation of spike generation; by contrast inhibitory input remained much smaller than excitatory input in neurons tuned to higher frequency, thus ensuring high-fidelity transmission. The tonotopic regulation of excitatory and inhibitory input optimized the output across frequencies and intensities, playing a fundamental role in the timing coding pathway in the auditory system.

\section{Introduction}

Nucleus magnocellularis (NM), the avian homolog of the mammalian anteroventral cochlear nucleus (AVCN), is the first central auditory brain region that conveys the temporal information of sound. NM neurons receive excitatory input from auditory nerve fibers (ANFs) and generate spikes at a specific phase of

\footnotetext{
Received May 16, 2019; revised 0ct. 27, 2019; accepted Nov. 4, 2019.

Author contributions: M.A.-Y., R.Y., and H.K. designed research; M.A.-Y. performed research; M.A.-Y., R.Y., and H.K. analyzed data; M.A.-Y., R.Y., and H.K. wrote the paper.

This work was supported by a Grant-in-aid from MEXT (18H02541 to H.K. and 16K07345 to R.Y.) and an Innovative Areas "Dynamic regulation of Brain Function by Scrap \& Build" (19H04747) Grant to H.K. M.A.-Y. was supported by a scholarship from MEXT. We thank Dr. L. 0. Trussell for editing the paper.

The authors declare no competing financial interests.

Correspondence should be addressed to Hiroshi Kuba at kuba@med.nagoya-u.ac.jp.

https://doi.org/10.1523/JNEUROSCI.1124-19.2019

Copyright $\odot 2020$ the authors
}

sound waves (Sullivan and Konishi, 1984; Warchol and Dallos, 1990), and send these phase-locked spikes bilaterally to coincident detectors in nucleus laminaris (NL) for sound localization (Hackett et al., 1982; Young and Rubel, 1983). Thus, the level and timing of NM signals are critical for accurate coincidence detection, and therefore must be tuned across sound frequency and intensity by multiple mechanisms.

One of these mechanisms is the tonotopic differentiation of excitatory input. NM neurons are tuned to a specific frequency of sound [the characteristic frequency (CF)] and arranged in an orderly manner within the nucleus, such that higher CFs are represented more rostromedially (Rubel and Parks, 1975). The number and size of excitatory inputs differ along the tonotopic axis (Parks and Rubel, 1978; Jhaveri and Morest, 1982; Carr and Boudreau, 1991; Köppl, 1994; Fukui and Ohmori, 2004). This 
ensures a precise output across frequencies, despite the fact that the fluctuation of spike timing (jitter) is larger in ANFs tuned to low-frequency sound (Köppl, 1997; Fukui et al., 2006). Low-CF neurons receive small bouton terminals from multiple ANFs, each of which causes a small response insufficient to generate a spike. Accordingly, the convergence of multiple inputs is required for spike generation, making the jitter smaller than that of ANFs in the low-CF neurons (Kuba and Ohmori, 2009). On the other hand, high-CF neurons receive one or a few end-bulb terminals, each of which causes a large response sufficient to generate a spike (Parks and Rubel, 1978; Trussell, 1999), allowing the high-CF neurons to match their jitter with that of ANFs.

Another mechanism is related to the inhibitory input. NM neurons receive GABAergic inhibitory inputs from the superior olivary nucleus (SON), which is in turn driven by ANFs primarily via nucleus angularis (NA), the other cochlear nucleus in the avian brainstem (Lachica et al., 1994; Yang et al., 1999; Burger et al., 2005). The effects of these inhibitory inputs have been studied in neurons with higher CF, presumably $>0.5 \mathrm{kHz}$, and showed that inhibition reduces the level and jitter of spike output specifically for intense sounds through an increase of a shunting conductance (Monsivais et al., 2000; Fukui et al., 2010; Howard and Rubel, 2010). However, it remains unclear how the balance between the excitatory and inhibitory inputs changes according to the activity of ANFs and how that balance shapes the output of NM. Importantly, it is expected that GABAergic shunting conductance is more efficient in suppressing spike generation in neurons receiving small (i.e., weaker) converging excitatory inputs than large high-fidelity inputs. Moreover, there is an increasing gradient of GABAergic terminals toward the low-CF region in NM (Code et al., 1989), raising the possibility that the excitatory and inhibitory balance is regulated differentially along the tonotopic axis in NM.

In this study, we developed a novel thick-slice preparation in which the entire excitatory and inhibitory circuit to NM was preserved, and examined the relationship between excitatory and inhibitory synaptic strength in NM neurons by stimulating ANFs. Amplitudes of EPSCs and IPSCs increased with an increase of stimulus intensity, but their dependence on intensity differed among the tonotopic regions; IPSCs in low-CF neurons appeared at an intensity similar to that of EPSCs and increased in parallel with EPSCs, whereas IPSCs in higher-CF neurons required a much higher intensity than EPSCs and appeared only at strong stimuli. We also found that the coupling of EPSCs and IPSCs in the low-CF neurons prevented the saturation of spike output, suggesting that the excitatory and inhibitory balance is strategically adjusted across tonotopic regions to ensure precise temporal coding for a broad range of intensities.

\section{Materials and Methods}

Animals. Chickens (Gallus domesticus) of either sex at post-hatch days $0-7$ were used in this study. The auditory system of these animals were almost mature at these ages, in terms of auditory threshold as well as synaptic and membrane properties of NM neurons (Saunders et al., 1973; Jhaveri and Morest, 1982; Code et al., 1989; Brenowitz and Trussell, 2001; Fukui and Ohmori, 2004). The care of experimental animals was in accordance with the regulations for animal research at Nagoya University and the experiments were approved by the institutional committee.

Slice preparation. Under deep anesthesia with ether, the chick was rapidly decapitated and the brainstem was isolated. ANFs were cut with microscissors at the orifice of the temporal bone, corresponding to a position $\sim 5 \mathrm{~mm}$ away from the brainstem. The brainstem was transferred to an oxygenated ice-cold high-glucose artificial CSF (HG-ACSF; concentrations in mu: $75 \mathrm{NaCl}, 2.5 \mathrm{KCl}, 26 \mathrm{NaHCO}_{3}, 1.25 \mathrm{NaH}_{2} \mathrm{PO}_{4}$, 100 glucose, $1 \mathrm{CaCl}_{2}, 3 \mathrm{MgCl}_{2}$, $\mathrm{pH}$ 7.4). After removing the membranous tissues, the brainstem was embedded in $3-4 \%$ agarose and glued to a slicing chamber with the caudal side up. The slicing chamber was filled with HG-ACSF and the brainstem was trimmed coronally to the level near the entrance of ANFs, where the outline of the caudal side of NM was visible. From this point, we made a thick coronal slice $(2-2.5 \mathrm{~mm})$ extending toward the rostral side (Fig. $1 A$ ). The thick slice was removed carefully from the agarose and incubated in HG-ACSF $\left(37^{\circ} \mathrm{C}\right)$ for $30-45$ min before use.

Tonotopic regions of NM. NM was divided into three tonotopic regions according to their position within the nucleus; low-CF region was defined as the caudo-lateral third, middle-CF region as the middle third, and high-CF region as the rostromedial third (Rubel and Parks, 1975; Fukui and Ohmori, 2004; Akter et al., 2018). As neurons in middle- and high-CF regions were qualitatively similar, they were combined and comparisons were made between low-CF and middle/high-CF regions. For recording from low-CF neurons, the tip of a recording pipette was positioned at the lateral half of NM within $200 \mu \mathrm{m}$ from the surface, whereas for middle/high-CF neurons, the tip was placed at the medial half of NM with a depth $>200 \mu \mathrm{m}$. We sometimes removed the caudal side of the thick slices (200-300 $\mu \mathrm{m}$ thickness) to increase accessibility to the middle/high-CF neurons.

Electrophysiology. Thick slices were transferred to the recording chamber caudal side up. For all experiments, the recording chamber was perfused with an oxygenated artificial CSF (ACSF; concentrations in mM: $125 \mathrm{NaCl}, 2.5 \mathrm{KCl}, 26 \mathrm{NaHCO}_{3}, 1.25 \mathrm{NaH}_{2} \mathrm{PO}_{4}, 17$ glucose, $2 \mathrm{CaCl}_{2}, 1$ $\mathrm{MgCl}_{2}, \mathrm{pH}$ 7.4). The extracellular solution was circulated by a peristaltic pump (ATTO) at a constant rate $(3-4 \mathrm{ml} / \mathrm{min})$. The recording temperature was maintained at $36-37^{\circ} \mathrm{C}$. Patch pipettes had a resistance of $4-6$ $\mathrm{M} \Omega$ (tip diameter of $1.5-2 \mu \mathrm{m}$ ) when filled with a $\mathrm{K}^{+}$-based solution (concentrations in mM: $113 \mathrm{~K}^{+}$-gluconate, $4.5 \mathrm{MgCl}_{2}, 0.1 \mathrm{EGTA}, 14$ Tris 2 -phosphocreatine, $4 \mathrm{Na}_{2}$-ATP, 0.3 Tris-GTP, 9 HEPES-KOH, $\mathrm{pH}$ 7.2) for current-clamp recording, or with a $\mathrm{Cs}^{+}$-based solution (concentrations in mM: $140 \mathrm{Cs}^{+}$-methanesulfonate, $20 \mathrm{CsCl}, 0.2 \mathrm{EGTA}, 10$ HEPES, pH 7.2) for recordings of EPSCs and IPSCs. Recordings of extracellular spikes were made using a pipette filled with the ACSF (тм: $125 \mathrm{NaCl}, 2.5 \mathrm{KCl}, 26 \mathrm{NaH}_{2} \mathrm{PO}_{4}, 17$ glucose, $2 \mathrm{CaCl}_{2}, 1 \mathrm{MgCl}_{2}$ ) under the cell-attached clamp, which preserves the intracellular $\mathrm{Cl}^{-}$environment. The recording pipette was advanced from the medial to the lateral side at an angle of $65^{\circ}$ from the horizontal with a $2 \mu \mathrm{m}$ step using a motorized micromanipulator (DMA-1511, Narishige) while applying positive pressure $(50-75 \mathrm{mmHg})$. A square wave voltage pulse $(5 \mathrm{mV}, 10 \mathrm{~ms}$ ) was continuously applied to monitor the pipette resistance. Upon a small increase in the resistance, the pipette was retracted $(6-10 \mu \mathrm{m})$ and then advanced again with a lower positive pressure (5-15 $\mathrm{mmHg}$ ). After a sufficient increase in the resistance, small negative pressure was applied to get a gigaohm seal. The whole-cell configuration was achieved by additional negative pressure. In some experiments, DNQX (60-80 $\mu \mathrm{M}$; Tocris Bioscience) and SR-95531 (100 $\mu \mathrm{M}$; Sigma-Aldrich) were applied to the bath to block excitatory and inhibitory transmission, respectively.

Electrical stimulation. EPSCs and polysynaptic IPSCs were induced in thick slices by stimulating ipsilateral ANFs with a bipolar tungsten electrode (75-125 $\mu \mathrm{m}$ thickness; Fig. $1 A$ ), which was inserted within the distal part of ANF bundle (Fig. $1 A$, red arrow). When the electrode was separated by $>4-5 \mathrm{~mm}$ from the lateral edge of NM it was not able to activate inhibitory fibers from SON to NM (see Results). Monosynaptic IPSCs in thick slices were induced by stimulating SON fibers with a bipolar tungsten electrode inserted deep into the slices at the ventrolateral side of NM, where SON fibers descend toward NM. Biphasic pulses, which minimized stimulus artifacts, were made with an electronic stimulator (SEN-7203, Nihon Kohden), and a single stimulus or a train of 20 stimuli (100,200, or $333 \mathrm{~Hz}$ ) were applied through an isolator (SS-202J, Nihon Kohden). The duration rather than the amplitude of the pulses was altered, as changing duration allowed us to adjust the stimulus intensity in discrete and reproducible increments in each experiment (Fig. $1 B)$. The amplitude of stimuli was initially set to induce reliable responses in each cell $(2-100 \mathrm{~V})$ and remained constant throughout experiments. When the rise time of EPSCs, which would reflect synchronized spike 
A
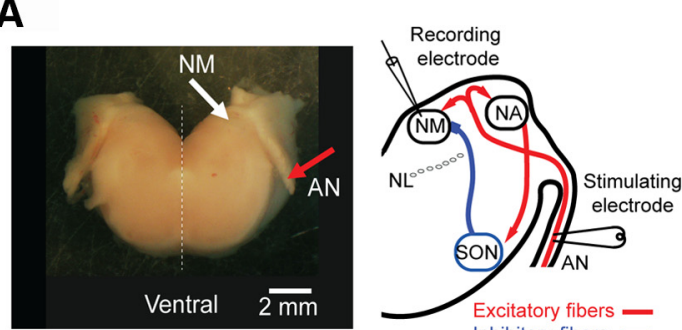

B

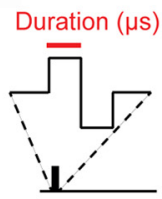

Stimulus intensity ( $\mu \mathrm{s})$

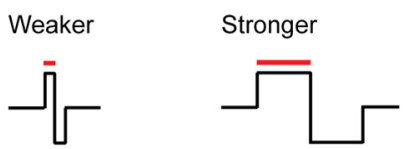

C
Low-CF

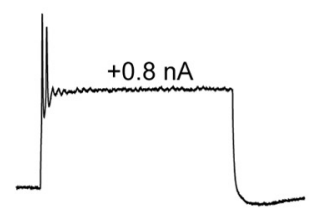

Excitatory fibers Inhibitory fibers -

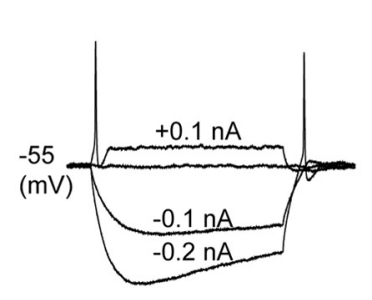

D
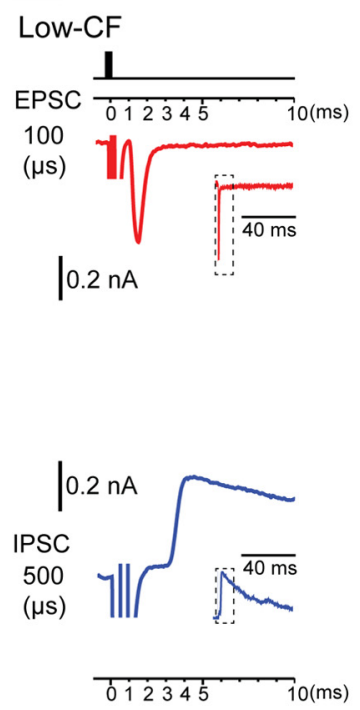

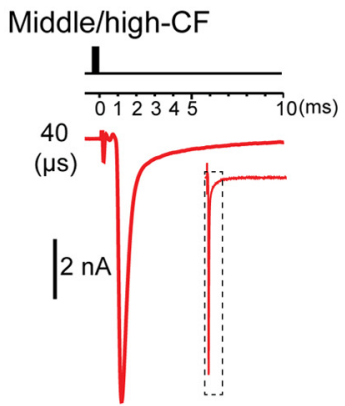

E

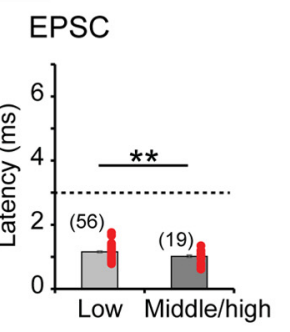

IPSC

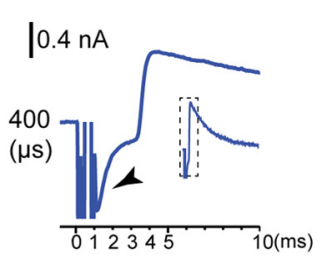

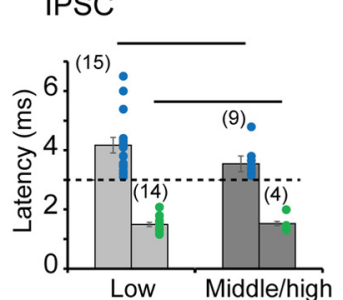

Middle/high-CF
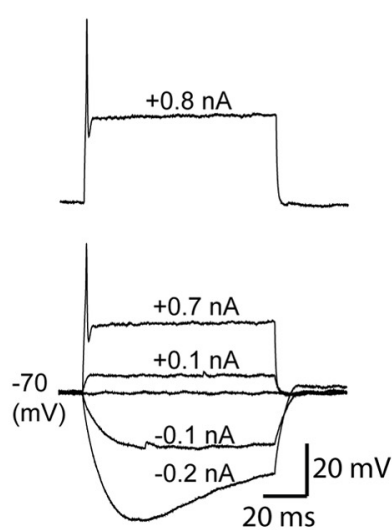

F
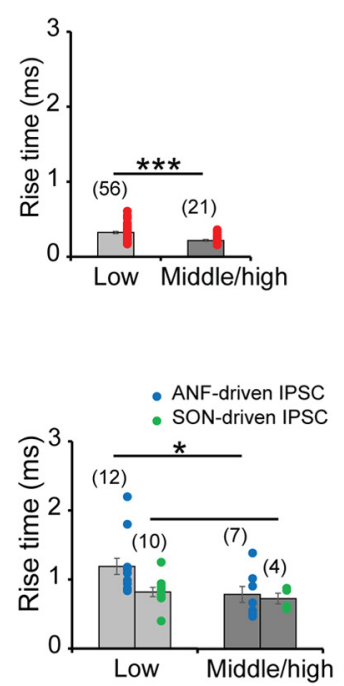

\section{G}
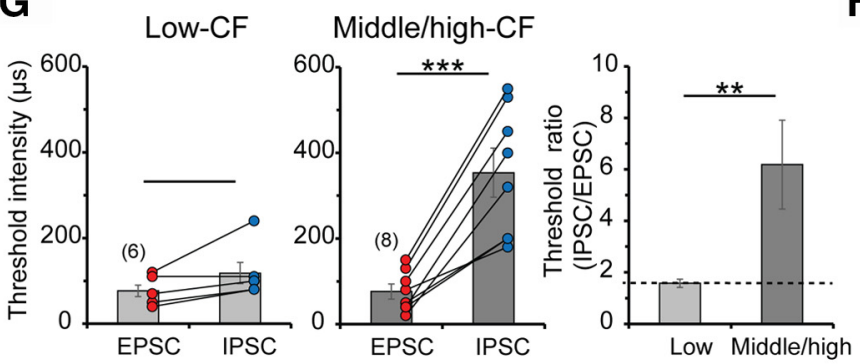

H

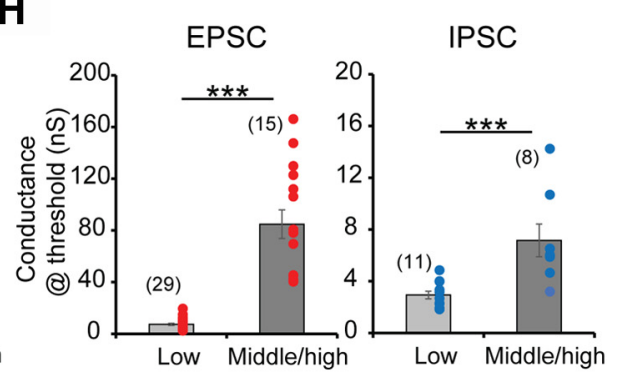

Figure 1. Monosynaptic EPSCs and polysynaptic IPSCs driven by ANFs. A, Thick-slice preparation of chicken brainstem (left), and excitatory and inhibitory pathways from ANFs to NM (right). The excitatory pathway is monosynaptic, whereas the inhibitory pathway is trisynaptic and mediated via NA and SON. A bipolar stimulating electrode was inserted into the auditory nerve at a position $>4-5 \mathrm{~mm}$ away from the lateral edge of NM (red arrow; see Materials and Methods), while postsynaptic responses were recorded from NM neurons (white arrow). $\boldsymbol{B}$, Biphasic electrical pulses were used to stimulate ANFs (left; see Materials and Methods). The stimulus intensity was adjusted by changing the duration of the pulse (red bar), where the intensity was weakened by decreasing the duration or strengthened by increasing the duration, whereas the amplitude was kept constant (right). $C$, Voltage responses of NM neurons in thick slices. Current pulses (80 ms) were applied to the soma between -0.2 and $0.8 \mathrm{nA}$. Both low-CF (left) and middle/high-CF (right) neurons generated only one or a few spikes at the onset of current injection, but low-CF neurons required less current for spike generation than middle/high-CF neurons ( 0.1 vs $0.7 \mathrm{nA}$, threshold current; Table 1), consistent with previous observations in thin slices (Fukui and 0hmori, 2004). D, EPSCs (top, red) and IPSCs (bottom, blue) in low-CF (left) and middle/high-CF (right) neurons. Currents were recorded in a neuron by switching holding potential between -50 and $0 \mathrm{mV}$, and ensemble-averaged from 5 to 7 traces. Inset showed traces in a longer time scale, and stimulus intensity was indicated at left. EPSCs were sometimes contaminated at $0 \mathrm{mV}$ as a small inward current before IPSCs in middle/high-CF neurons (black arrowhead, bottom right), presumably due to their huge conductance and a slight shift of holding potential by the series resistance (see Materials and Methods). $\boldsymbol{E}$, $\boldsymbol{F}$, Latency $(\boldsymbol{E})$ and rise time $(\boldsymbol{F})$ of EPSCS (top, red dots) and IPSCS (bottom, blue dots). Monosynaptic IPSCs induced by stimulating SON fibers were also plotted (bottom, green dots). The latency of ANF-driven IPSCs was always longer than 3 ms (broken lines), whereas that of EPSCs and SON-driven IPSCs was $<3$ ms. G, Threshold intensity to induce synaptic currents. Threshold intensity was similar between EPSCs and IPSCs in low-CF neurons (left), whereas it was four times higher for IPSCs in middle/high-CF neurons (middle), making the ratio between IPSCs and EPSCs larger in those neurons (right). $\boldsymbol{H}$, Conductances at threshold intensity for EPSCS (left) and IPSCS (right). Numbers in parenthesis are the number of cells. ${ }^{*} p<0.05,{ }^{* *} p<0.01,{ }^{* * *} p<0.001$. 
induction among ANFs, was evaluated in low-CF neurons in thin slices, the rise time increased only slightly during an increase of the duration; the extent of increase was $<0.2 \mathrm{~ms}(0.13 \pm 0.05 \mathrm{~ms})$, and was not different from that during an increase of the stimulus amplitude $(0.1 \pm 0.1 \mathrm{~ms}$; $n=7, p=0.5$ ).

Data acquisition and analysis. Recordings were performed with a patch-clamp amplifier (EPC8, HEKA Elektronik). Output signals were low-pass filtered at $3 \mathrm{kHz}$, sampled at $50 \mathrm{kHz}$ (BNC-2090A, National Instruments), stored, and analyzed on a personal computer with AxoGraph X (AxoGraph Scientific). Voltages were corrected for a $10 \mathrm{mV}$ liquid junction potential. Series resistance was $<10 \mathrm{M} \Omega$ and was not compensated. Threshold current of spikes was measured as the minimum current required for spike induction, threshold voltage as the voltage corresponding to an inflection point in the first time derivative of voltage, and amplitude as the voltage between the threshold and the peak. Reversal potentials of EPSCs and IPSCs were $0 \mathrm{mV}$ and $-50 \mathrm{mV}$, respectively, which allowed us to record both synaptic currents in the same neuron with a substantial driving force by switching the holding potential between -50 and $0 \mathrm{mV}$. A small EPSC appeared at $0 \mathrm{mV}$ before an IPSC in some middle/high-CF neurons ( 4 of 12 cells), implying a slight shift of the holding voltage due to the series resistance. Nevertheless, the EPSCs were smaller by $\sim 25$ times at $0 \mathrm{mV}$ than at $-50 \mathrm{mV}$, implying that the shift would be $\sim 2 \mathrm{mV}$ and the underestimation of IPSCs would be only $\sim 4 \%$. Latency of synaptic currents was defined as the period between the onset of the stimulus artifact and $10 \%$ of the current peak, and rise time as the period between 10 and $90 \%$ of the peak. Threshold intensity of synaptic currents was defined as the minimum intensity (i.e., the shortest pulse duration) of generating synaptic responses in every trial. The paired-pulse ratio (PPR) was calculated from the amplitudes of the first and the second stimuli, and the amplitudes were measured between the onset and the peak at the stimuli. Synaptic conductance was measured between the initial baseline and the peak at each stimulus. Firing probability of extracellular spikes was defined as the total number of spikes divided by the total number of stimuli, and calculated from 10 to 13 traces. Threshold intensity of the extracellular spike was defined as the intensity at $10 \%$ of firing probability, saturating intensity as the intensity reaching $100 \%$ of probability, and dynamic range as the difference between the threshold and saturating intensities. Spike jitter was defined as the SD of latency peak, and it was calculated from $>10$ traces.

Computational model. Neuronal modeling and simulation were performed with NEURON 7.6, as described previously (Kuba and Ohmori, 2009). The model of low-CF NM neurons consisted of multiple sections: a soma ( $20 \mu \mathrm{m}$ in length and diameter) and an axon with an axon hillock (10 $\mu \mathrm{m}$ length), an axon initial segment (20 $\mu \mathrm{m}$ length), 10 myelinated internodes, and 10 nodes of Ranvier. The $\mathrm{Na}^{+}$current (Rothman and Manis, 2003), and low- and high-voltage-activated $\mathrm{K}^{+}$currents (Klva and Khva; Rathouz and Trussell, 1998) followed the web-accessible Model DB (Hines et al., 2004). The $\mathrm{V}_{1 / 2}$ and slope factor for activation were -58 and $10 \mathrm{mV}$ for the Klva current, and -19 and $9 \mathrm{mV}$ for the Khva current, respectively. The $\mathrm{Na}^{+}$, Klva, and Khva currents were incorporated into the soma, the axon initial segment, and the nodes; gNa, gKlva, and gKhva were 100,60 , and $60 \mathrm{pS} / \mu \mathrm{m}^{2}$ in the soma, and 14,000 $\mathrm{pS} / \mu \mathrm{m}^{2}, 180$, and $180 \mathrm{pS} / \mu \mathrm{m}^{2}$ in the axon initial segment and the nodes, respectively. The Khva current was also included at the axon hillock (180 $\left.\mathrm{pS} / \mu \mathrm{m}^{2}\right)$. The temperature was $40^{\circ} \mathrm{C}$. The time step of the calculation was $12.5 \mu$ s. Excitatory input consisted of multiple unitary EPSC conductances (uEPSGs), and the input timing of each uEPSG varied randomly according to the normal distribution with a SD of $0.24 \mathrm{~ms}$. Individual uEPSG had a profile of $\alpha$ function $\left[=\mathrm{g}_{\text {syn }} \cdot t / \tau_{\text {syn }} \cdot \exp (1-\right.$ $\left.t / \tau_{\text {syn }}\right)$ ], time constant $\left(\tau_{\text {syn }}\right)$ of $0.3 \mathrm{~ms}$, and amplitude of $4 \mathrm{nS}$, corresponding to those in low-CF NM neurons (Fukui and Ohmori, 2004), unless otherwise stated. Inhibitory input consisted of multiple unitary IPSC conductances (uIPSGs), each of which was a tonic conductance with the size and the number the same as those of uEPSG. The reversal potential of IPSCs was set at $-60 \mathrm{mV}$, which was measured in low-CF neurons (unpublished observations; for more depolarized reversal potential in middle/high-CF NM neurons, see Hyson et al., 1995; Lu and Trussell, 2001; Monsivais and Rubel, 2001). When the reversal potential
Table 1. Membrane and spike parameters of NM neurons in the thick slice preparation

\begin{tabular}{lcc}
\hline & Low CF & Middle/high CF \\
\hline Resting potential, $\mathrm{mV}$ & $-61.2 \pm 1.9$ & $-69.6 \pm 0.5^{* * *}$ \\
Input resistance, $\mathrm{M} \Omega$ & $164.1 \pm 16.9$ & $62.9 \pm 10.2^{* * *}$ \\
Membrane time constant, $\mathrm{ms}$ & $10.7 \pm 2.2$ & $3.5 \pm 1.1^{* *}$ \\
Threshold current, $\mathrm{pA}$ & $75 \pm 17$ & $640 \pm 164^{* *}$ \\
Threshold potential, $\mathrm{mV}$ & $-53.1 \pm 3.1$ & $-41.6 \pm 5.9$ \\
Spike amplitude, $\mathrm{mV}$ & $43.1 \pm 3.1$ & $18.8 \pm 3.8^{* * *}$ \\
\hline
\end{tabular}

Membrane and spike parameters were measured in low-CF $(n=6)$ and middle/high-CF $(n=5)$ neurons by applying somatic current injection under current clamp (see Materials and Methods).

${ }^{* *} p<0.01,{ }^{* * *} p<0.001$ between low-CF and middle/high-CF neurons.

of IPSCs was depolarized, inhibitory effects became smaller. Firing probability was calculated from 50 traces.

Statistics. Data were presented as a mean \pm SE $(n=$ number of cells $)$. Statistical evaluation was made with paired or unpaired $t$ test for comparison of two groups.

\section{Results}

\section{NM neurons in the thick-slice preparation}

Using the thick-slice preparation, we recorded voltage responses of current-clamped NM neurons to somatic injection of rectangular current pulses through the recording pipette (Fig. 1A,C). Basic membrane parameters varied with tonotopic regions (Table 1), such that the resting potential was more positive and the input resistance and membrane time constant were larger in low-CF neurons than in middle/high-CF neurons. Spike parameters were also different between the tonotopic regions (Table 1): the threshold current was smaller, the threshold potential was lower, and the amplitude was larger in the low-CF neurons. In addition, the threshold current generated a single spike at stimulus onset in both neurons, whereas a larger current generated more than one spike specifically in low-CF neurons ( $0.8 \mathrm{nA}$; Fig. $1 C)$. These observations were compatible with those in conventional thin slices (Fukui and Ohmori, 2004; Akter et al., 2018), confirming that the condition of cells was maintained and tonotopic regions were appropriately estimated in the present recordings.

\section{Polysynaptic IPSCs were driven by stimulation of ANFs}

We stimulated ANFs with a bipolar tungsten electrode placed at 4-5 $\mathrm{mm}$ away from the recording position and recorded synaptic currents in NM neurons under the voltage clamp (Fig. 1A) while adjusting stimulus intensity by changing the pulse duration at a constant amplitude (Fig. 1B; see Materials and Methods). EPSCs and IPSCs were isolated in the neurons by switching holding potential between -50 and $0 \mathrm{mV}$ with a pipette filled with a $\mathrm{Cs}^{+}$-based solution (see Materials and Methods). In both low-CF and middle/high-CF neurons, EPSCs appeared as inward currents at $-50 \mathrm{mV}$ (Fig. $1 D$, top, red traces), whereas IPSCs appeared as outward currents at $0 \mathrm{mV}$ (Fig. $1 D$, bottom, blue traces). The latency of EPSCs was $<2 \mathrm{~ms}$ in both areas (Fig. 1E, top, red dots), reflecting the monosynaptic innervation of ANFs to NM. On the other hand, the latency of IPSCs was always $>3 \mathrm{~ms}$ (Fig. 1E, bottom, blue dots), and it was apparently separated from that of monosynaptic IPSCs $(<2 \mathrm{~ms}$, green dots; see Materials and Methods), suggesting that SON fibers were not directly activated during the stimulation of ANFs. In addition, because NA is located more rostrally than NM, NA fibers are further from the stimulating electrode and hence would have a higher threshold than SON fibers projecting to NM. Thus, the present stimulation procedure would induce trisynaptic IPSCs via the activation of ANFs with little effects on fibers from NA and SON. Contribu- 
tions of local interneurons around NM and NL (von Bartheld et al., 1989; Yamada et al., 2013) to the IPSCs would be small, because IPSCs were totally abolished when SON was removed by cutting the ventral part of the slice (data not shown).

The decay phase of IPSCs was $40-50$ times slower than that of EPSCs, as observed in thin slices (Zhang and Trussell, 1994; Kuo et al., 2009), and did not differ between low-CF and middle/ high-CF neurons; decay time constant of EPSCs was $0.58 \pm 0.02$ $\mathrm{ms}(n=51)$ and $0.49 \pm 0.04 \mathrm{~ms}(n=18)$ in low-CF and middle/ high-CF neurons, respectively ( $p=0.05$ ), and that of IPSCs was $22.2 \pm 6.6 \mathrm{~ms}(n=5)$ and $17.0 \pm 6.5 \mathrm{~ms}(n=3)$, respectively $(p=0.3)$. In contrast, the rising phase was slightly slower in the low-CF neurons for both EPSCs and IPSCs (Fig. $1 F$ ). This could be related to the fact that low-CF neurons are innervated by multiple ANFs, activation of which is likely to cause a variation in the timing of spike induction among the fibers.

We examined the minimum conductance of EPSCs and IPSCs driven by ANFs at the threshold intensity defined as the shortest pulse duration that induced responses (Fig. 1G,H; see Materials and Methods). The threshold intensity did not differ between EPSCs and IPSCs in low-CF neurons (Fig. 1G, left), whereas it was 3-4 times higher for IPSCs than for EPSCs in middle/ high-CF neurons (middle), making the relative threshold of IPSCs to EPSCs larger in middle/high-CF neurons (right). The conductance of EPSCs at the threshold intensity was $\sim 10$ times smaller in low-CF neurons than in middle/high-CF neurons (Fig. $1 H$, left), whereas that of IPSCs was $\sim 2-3$ times smaller in the low-CF neurons (right). These data showed that the inhibitory input was comparable to the excitatory input in both size and threshold in the low-CF neurons, whereas it was small and required a stronger stimulus intensity in the middle/ high-CF neurons.

\section{Polysynaptic IPSCs temporally summated during a stimulus train}

We examined EPSCs and IPSCs in response to a train of 20 stimuli at $200 \mathrm{~Hz}$ in thick slices (Fig. 2). EPSC amplitudes underwent strong depression during the train and the extent of depression was larger in low-CF neurons; PPR was smaller by $30 \%$, and conductance calculated at $18-20$ th stimuli was lower by $40 \%$ in low-CF neurons than in middle/high-CF neurons (Fig. 2A, $B$, top, $C$; see Materials and Methods), in agreement with a previous report (Oline and Burger, 2014). IPSCs also showed strong depression during the train, whereas the extent did not differ between neurons in different CF classes (Fig. 2A,B, bottom). Importantly, however, the decay kinetics of IPSCs was slow, allowing the IPSCs to summate temporally and produce a plateau current during the train. As a result, the conductance of IPSCs after 18-20th stimuli was larger than the conductance at the first stimulus (Fig. 2D). Thus, the excitatory conductance became smaller, and the inhibitory conductance became larger, during the train, suggesting that an impact of inhibitory input on neuronal output would increase as the train progressed. This effect was most prominent in low-CF neurons due to the depression of their EPSCs.

This depression of EPSCs became more pronounced as stimulus frequency increased, and the extent of depression was similar in both CF regions: the conductance of EPSCs at 18-20th stimuli decreased by 50 and $25 \%$ in low-CF and middle/high-CF neurons, respectively, between 100 and $333 \mathrm{~Hz}$ (Fig. 2F). Depression of IPSCs was also augmented at higher stimulus frequencies. Indeed, PPR decreased by 65 and $60 \%$ in low-CF and middle/ high-CF neurons, respectively, between 100 and $200 \mathrm{~Hz}$, whereas
PPR was not calculated at $333 \mathrm{~Hz}$ because stimulus artifacts overlapped the rising phase of IPSCs at the frequency (data not shown). On the other hand, the conductance of IPSCs at 18-20th stimuli either did not change or became even larger at higher frequencies in both CF regions: the increase was 28 and $4 \%$ in low-CF and middle/high-CF neurons, respectively, between 100 and $333 \mathrm{~Hz}$ (Fig. 2G). This occurred because the decay kinetics of IPSCs was slow and the temporal summation of IPSCs became more pronounced at higher frequencies (Roberts et al., 2014).

IPSCs driven by stimulation of SON fibers showed strong depression, and the extent of depression and summation was similar to that of polysynaptic IPSCs driven by ANF stimulation (Fig. $2 B, E$ ). In addition, the conductance at 18-20th stimuli did not change with stimulus frequency (Fig. $2 \mathrm{H}$ ), suggesting that the pattern of polysynaptic inhibitory input would reflect that of monosynaptic transmission of SON fibers in NM.

\section{Polysynaptic IPSCs increased linearly with EPSCs in low-CF neurons}

We then examined the balance between the excitatory and inhibitory conductances for a given number of ANFs, by elevating the stimulus intensity via a stepwise increase of pulse duration $(10 \mu \mathrm{s})$ while switching holding potential between -50 and $0 \mathrm{mV}$ (Fig. 3; see Materials and Methods). In low-CF neurons, EPSCs increased gradually with the stimulus intensity (Fig. $3 A$ ), which was consistent with convergence of multiple ANFs onto each low-CF neuron (Fukui and Ohmori, 2004). IPSCs also increased in a similar manner; they appeared with an intensity just above the threshold intensity of EPSCs ( $240 \mu \mathrm{s})$ and increased in parallel with EPSCs $(300-450 \mu \mathrm{s})$. We plotted the synaptic conductances from a population of cells against the intensity for the first stimulus (Fig. 3B, left) and the 18-20th stimuli (right). The conductances increased with the intensity for both EPSCs (red) and IPSCs (blue) at the 18-20th stimuli (right), whereas the dependence was rather weak for IPSCs at the first stimulus (left). In consequence, the excitatory and inhibitory conductances showed a strong positive correlation, and the tendency was more prominent at the $18-20$ th stimuli (Fig. $3 C$, right) than at the first stimulus (left).

In middle/high-CF neurons, EPSCs showed one or a few large steps with increase in intensity (Fig. 3B), reflecting the convergence of a small number of strong inputs to each cell, accounting for their high-fidelity transmission (Fukui and Ohmori, 2004). Importantly, IPSCs were not elicited even at a saturating intensity for EPSCs $(120 \mu \mathrm{s})$ and appeared only when extremely intense stimuli were applied (>320 $\mu$ s). In addition, a further elevation of stimulus amplitude could not increase IPSCs (data not shown), confirming that IPSCs required stronger stimuli for induction compared with EPSCs. Indeed, the threshold intensity for IPSCs was higher than that for EPSCs in all the cells, and most cells could not show more than one step within the intensity range of present stimuli (up to $600 \mu$ s; Figs. 3B, 1). Accordingly, the inhibitory conductance remained smaller than the excitatory conductance for both the first and the 18-20th stimuli in these neurons (Fig. 3 C). Thus, the inhibitory input in low-CF neurons was balanced with the excitatory input for the range of applied intensity particularly at the later part of the train, whereas that in middle/high-CF neurons was mostly dominated by the excitatory input, suggestive of a difference in the impact of inhibitory input on the neuronal output between the neurons. 
A
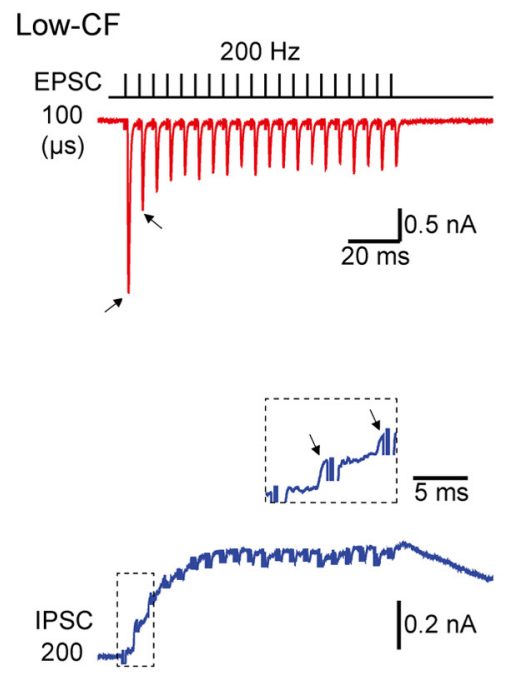

C

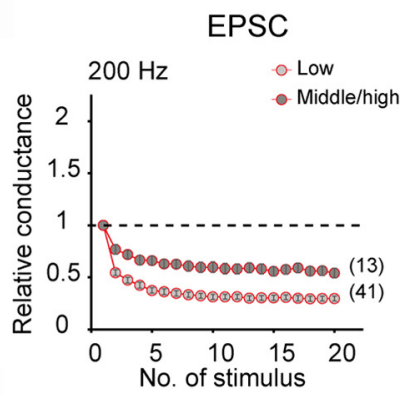

F

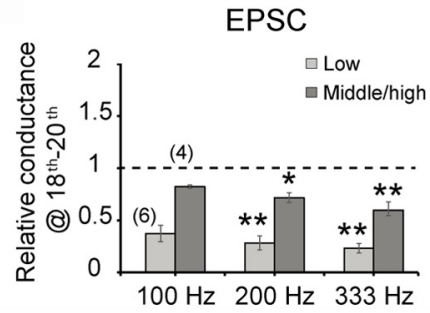

Middle/high-CF

$200 \mathrm{~Hz}$
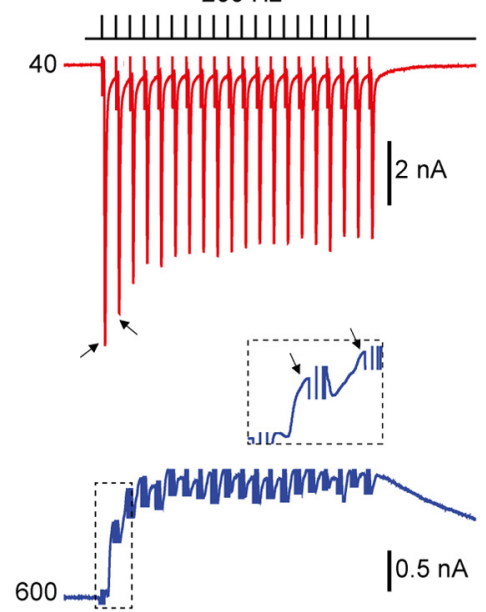

D

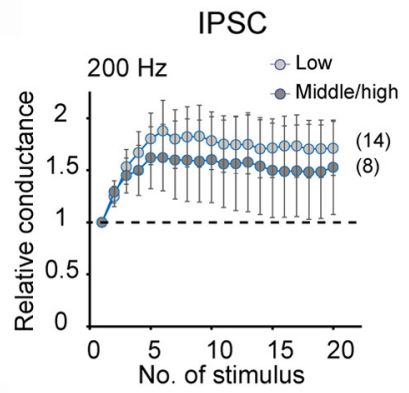

IPSC

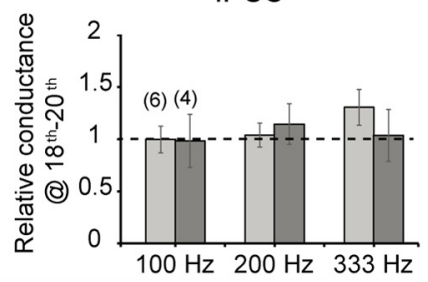

B

\section{EPSC}
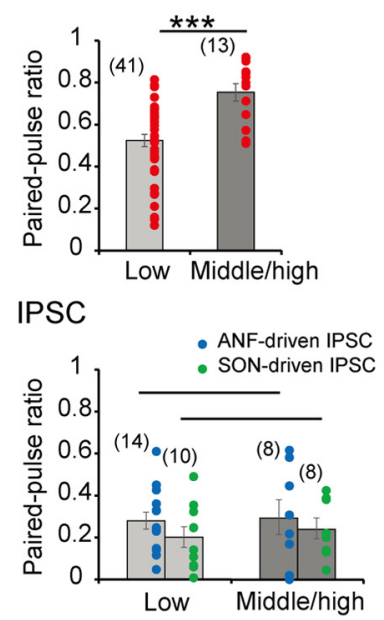

E

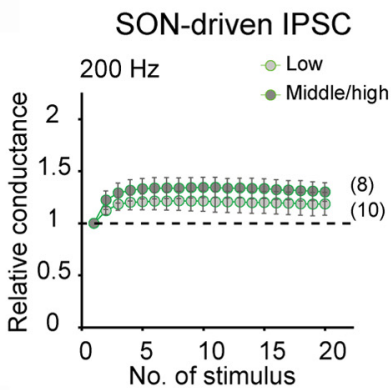

H

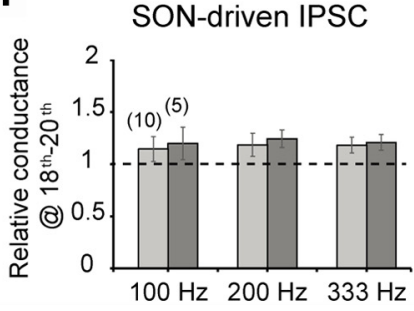

Figure 2. Temporal summation of polysynaptic IPSCS. A, EPSCs and IPSCs during a train of stimuli (20 pulses, $200 \mathrm{~Hz})$. Currents were ensemble-averaged from 5 to 7 traces. IPSCs at the initial two stimuli are expanded in the box, and arrows indicate the peak at each stimulus. Both currents showed strong synaptic depression, although IPSCS, which have slow decay kinetics, temporally summated leading to a plateau current. B, PPR of EPSCS (top, red dots) and IPSCS (bottom, blue dots) from the second and the first responses in the train. PPR of monosynaptic IPSCS driven by SON fibers is also shown (green dots). $\boldsymbol{C}-\boldsymbol{E}$, Synaptic conductance relative to the first stimulus for EPSCS (C), IPSCS (D), and SON-driven IPSCs (E). Relative conductance reached a steady level at the later part of the train, and the level was $<1$ for EPSCS, whereas it was similar to or $>1$ for both polysynaptic and monosynaptic IPSCs. $\boldsymbol{F}-\boldsymbol{H}$, Relative conductance at the 18th-20th stimuli during trains at 100, 200, and $333 \mathrm{~Hz}$ for EPSCS $(\boldsymbol{F})$, IPSCS $(\boldsymbol{G})$, and SON-driven IPSCS $(\boldsymbol{H})$. Values were averages of the $18-20$ th stimuli from 5 to 7 traces. Numbers in parenthesis are the number of cells. ${ }^{*} p<$ $0.05,{ }^{* *} p<0.01,{ }^{* * *} p<0.001$.

\section{Graded increase in spike responses with intensity in low-CF neurons}

The input/output relationship of NM neurons was examined in thick slices (Fig. 4). We stimulated ANFs with a train of 20 stimuli at $200 \mathrm{~Hz}$ and recorded extracellular spikes under the cellattached configuration while increasing pulse duration in a stepwise fashion (10 $\mu$ s; see Materials and Methods). The spikes showed a biphasic shape (Fig. $4 A$, inset) and had a latency of $1.5 \pm 0.1 \mathrm{~ms}(n=22)$ and $1.3 \pm 0.1 \mathrm{~ms}(n=7)$ for low-CF and middle/high-CF neurons, respectively. In the low-CF neurons, patterns of spike responses changed in time- and intensitydependent manners; spikes were restricted to the initial part of the train when a weak intensity $(80 \mu \mathrm{s})$ was used, although spikes also occurred later in the train with larger stimuli $(250 \mu \mathrm{s})$, reaching a $100 \%$ fidelity with the strongest $(400 \mu \mathrm{s})$ stimuli (Fig. $4 A$, left). The time- and intensity-dependences appear in part because the synaptic depression of EPSCs prevented spike generation during the later part of the train, but this effect was overcome by an increase of EPSC amplitude at the strong intensity (Fig. 3A, left). Consistent with this idea, spike jitter increased later in the train, and was reduced with higher intensity stimuli (Fig. 4A, inset). In middle/high-CF neurons, on the other hand, spikes persisted throughout the train, even at the lowest intensity ( $20 \mu \mathrm{s}$; Fig. $4 A$, right). In addition, the spike jitter remained small during the train (Fig. $4 A$, inset), as expected given the large unitary EPSCs in the neurons (Figs. 1-3). We plotted the firing probability versus the intensity for a group of cells in Figure $4 B$, showing that the firing probability increased gradually in the low-CF neurons (left), whereas it increased abruptly to reach a saturating level in the middle/high-CF neurons (right). 
A

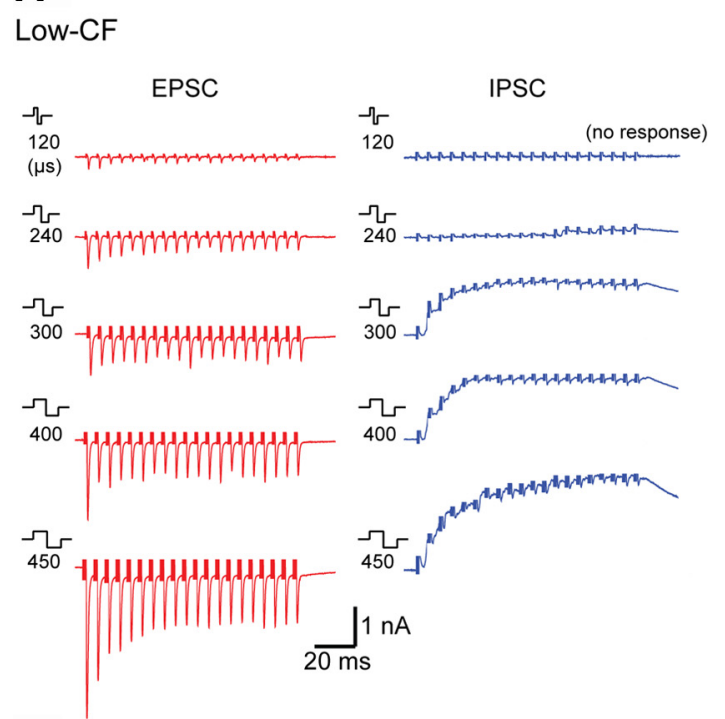

\section{Middle/high-CF}

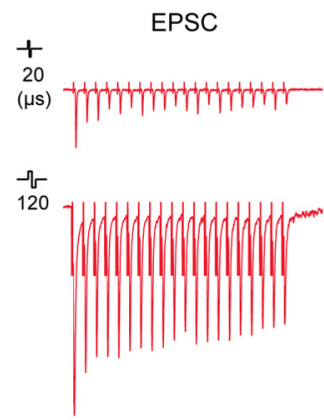

B
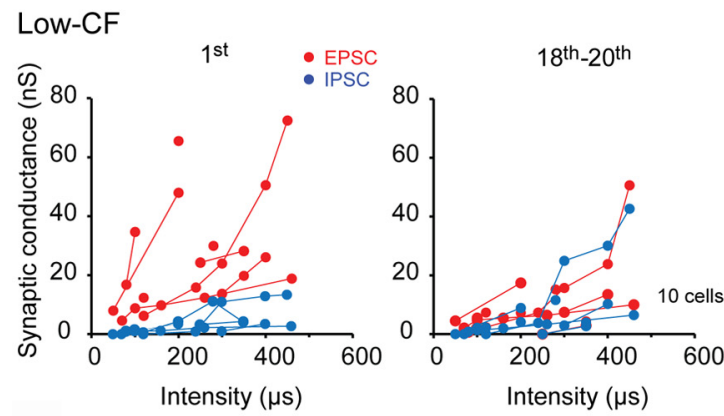

C
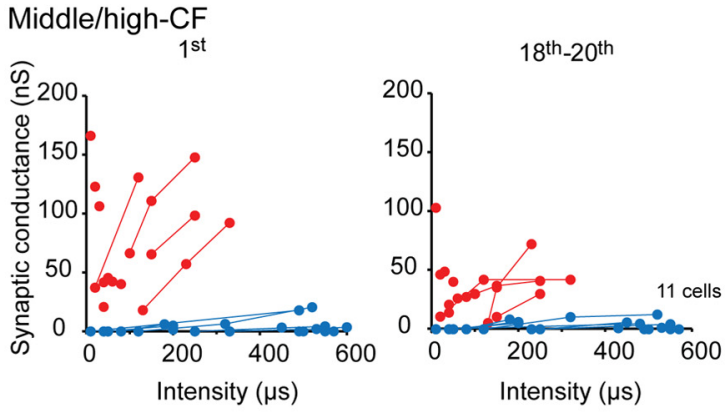

Low-CF
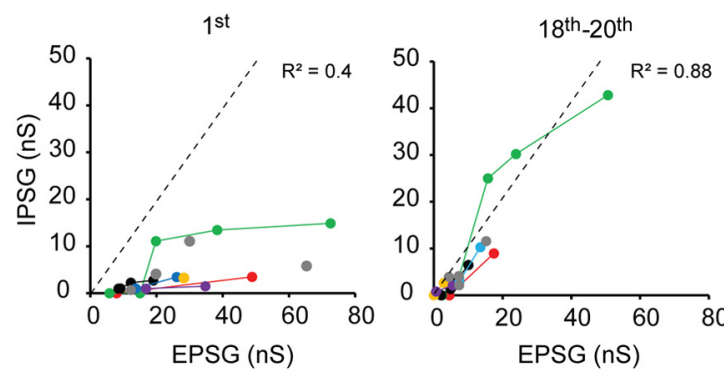

Middle/high-CF
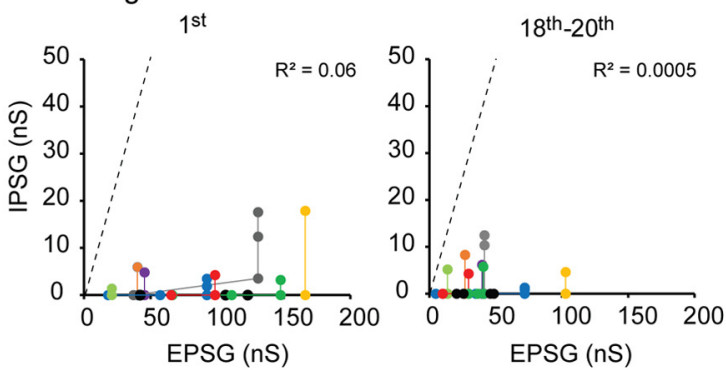

Figure 3. Polysynaptic IPSCs increased in parallel with EPSCs in low-CF neurons. A, EPSCs and IPSCs during a train of stimuli (20 pulses, $200 \mathrm{~Hz}$ ) of different intensities. The intensities were altered by changing the duration of pulses (in microseconds; left of each trace), whereas the amplitude of pulses was kept constant. Low-CF (left) and middle/high-CF (right) neurons. Currents were ensemble-averaged from 5 to 7 traces. Note that EPSCs and IPSCs appeared at a similar intensity and both increased gradually with intensity in the low-CF neuron, whereas IPSCs showed higher threshold and were far smaller than EPSCs even with an intense stimulus (520 $\mu$ s) in the middle/high-CF neuron. B, Relationship between synaptic conductance and intensity for EPSCs (red) and IPSCS (blue) at the first (left) and the 18th-20th (right) stimuli. Symbols connected by lines represent the same cells. Synaptic conductance was larger for EPSCs than for IPSCs at the first stimulus in both low-CF and middle/high-CF neurons, whereas it was almost overlapping at the 18-20th stimuli in low-CF neurons. C, Relationship between excitatory (EPSG) and inhibitory (IPSG) synaptic conductances from $B$. Both conductances were strongly correlated in low-CF neurons particularly at the 18-20th stimuli, whereas they were not correlated in middle/high-CF neurons. Different colors represent individual cells, and gray symbols in low-CF groups indicate cells in which conductances were measured at a single intensity. Black symbols in middle/high-CF groups correspond to cells in which IPSCs were not induced even with the maximum stimulus $(600 \mu \mathrm{s}, 100 \mathrm{~V})$. The absence of IPSCs likely does not result from a generalized deterioration of the inhibitory pathway because IPSCS were still elicited with a weaker stimulus $(143+32 \mu s, n=4)$ in low-CF neurons in the same preparation. Numbers in parenthesis are the number of cells.

Correspondingly, the low-CF neurons showed a higher saturating intensity (Fig. $4 D$ ) and a wider range of intensity for spike responses (Fig. 4E, dynamic range) than the middle/ high-CF neurons. Notably, the jitter of spikes was larger in the low-CF neurons (Fig. 4F), and the threshold intensity to induce spikes was also elevated in the neurons (Fig. 4C). Because the threshold intensity of EPSCs was similar between the neurons and the postsynaptic membrane excitability was even higher for the low-CF neurons (Fig. 1C; Table 1), the higher threshold intensity for the spike induction in the low-CF neurons is attributed to the substantial inhibitory input in the neurons. 
A

Low-CF
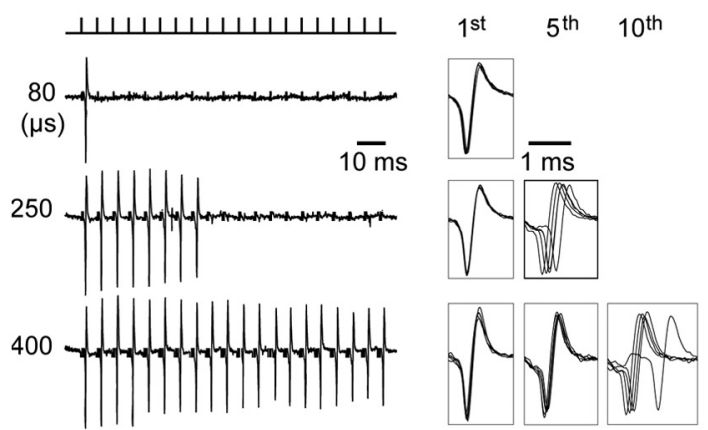

B

Low-CF

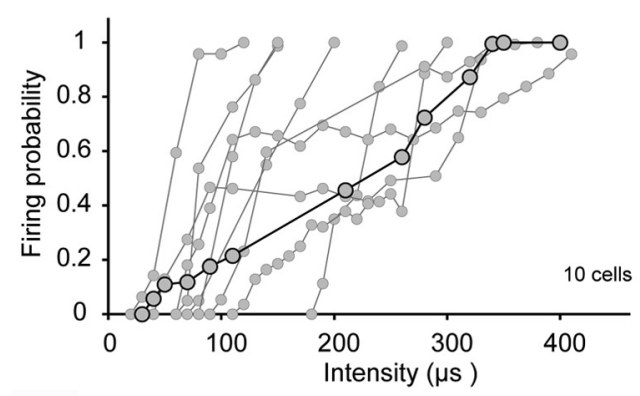

C

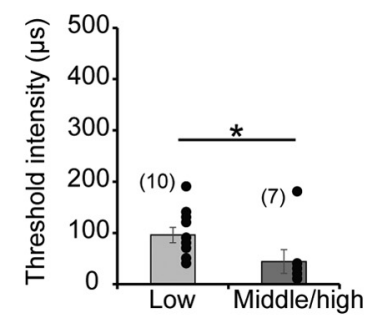

D

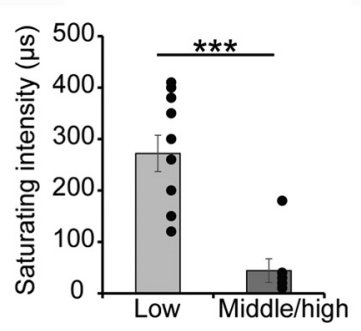

Middle/high-CF
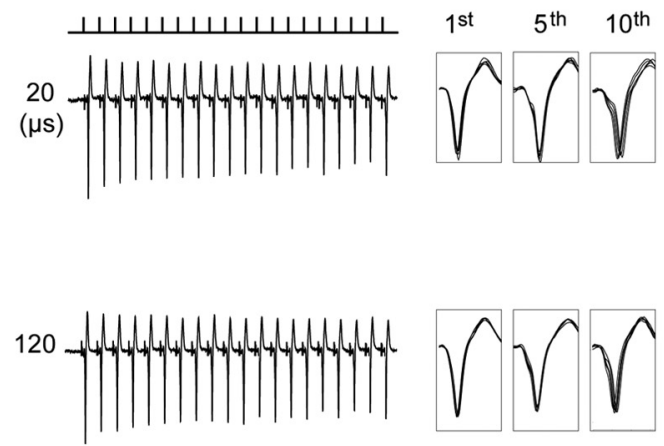

Middle/high-CF

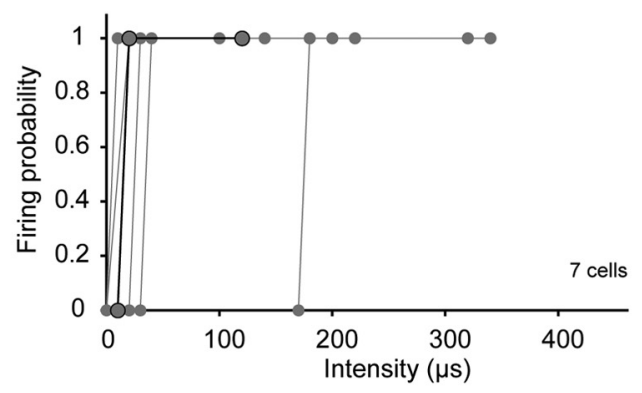

E

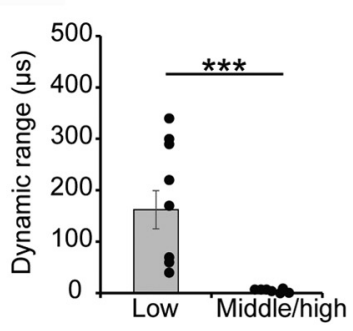

F

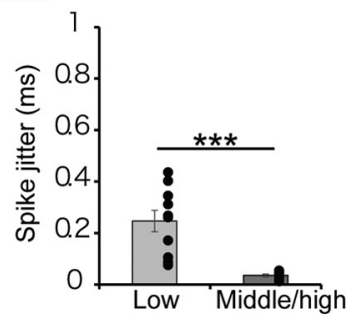

Figure 4. Spike output increased with ANF stimulus intensity in low-CF neurons. A, Spike output in response to a train of stimuli (20 pulses, $200 \mathrm{~Hz})$ of different intensities recorded under cell-attached condition. Low-CF (left) and middle/high-CF (right) neurons. Five traces were superimposed and spikes at corresponding stimuli were expanded (box). The number of spikes increased with intensity toward the later part in low-CF neurons, whereas the number reached the maximum even at the minimum intensity (20 $\mu \mathrm{s})$ in middle/high-CF neuron. Spike jitter became larger toward the later part and decreased with intensity in low-CF neurons. $\boldsymbol{B}$, firing probability was plotted against intensity. Symbols connected by lines represent the same cells. Firing probability was calculated from 10 to 13 traces at each intensity. $\boldsymbol{C}-\boldsymbol{F}$, Threshold intensity $(\boldsymbol{C}$, saturating intensity $(\boldsymbol{D})$, dynamic range $(\boldsymbol{E})$, and spike jitter $(\boldsymbol{F})$. Spike jitter was calculated at saturating intensity from all the spikes during the train. All the parameters were larger in low-CF neurons than in middle/high-CF neurons. Numbers in parenthesis are the number of cells. ${ }^{*} p<0.05,{ }^{* * *} p<0.001$.

\section{Polysynaptic IPSCs expanded the dynamic range of the output in low-CF neurons}

Given these differences between the ranges of stimuli that elicit inhibitory responses in different CF regions, we next examined the differential effects of blocking inhibitory inputs on spike generation by applying a $\mathrm{GABA}_{\mathrm{A}}$ receptor antagonist (SR-95531; 100 $\mu \mathrm{M})$. Before application of the drug, only a few spikes were generated in low-CF neurons at the onset for an intensity just above threshold (Fig. 5A, left, blue traces; 80 and $100 \mu \mathrm{s}$ ). SR-95531 enhanced spike responses and the number reached the maximum (20 spikes) at $100 \mu \mathrm{s}$ (Fig. 5A, right, red traces). Accordingly, SR-95531 caused a prominent leftward shift in the intensity dependence of firing probability in the neuron; it lowered both the threshold intensity (single arrowheads) from 60 to $40 \mu$ s and the saturating intensity (double arrowheads) from 300 to $100 \mu \mathrm{s}$, narrowing the dynamic range by $180 \mu \mathrm{s}$ (Fig. $5 B$ ). This pattern was consistent across neurons, and the dynamic range was $40 \pm$
$6 \%$ of control after SR-95531 ( $n=6, p=0.002$; Fig. $5 D-F)$, suggesting that the inhibitory input strongly suppressed spike generation in the low-CF neurons even at threshold intensity (Figs. 1, 4). SR-95531 shortened spike latency from $1.5 \pm 0.1 \mathrm{~ms}$ to $1.3 \pm 0.1 \mathrm{~ms}(n=6, p=0.002)$. In addition, SR-95531 decreased the spike jitter (Fig. $5 G$ ), presumably due to an increase of EPSP amplitude after a reduction of shunting conductance via a blockade of postsynaptic $\mathrm{GABA}_{\mathrm{A}}$ receptors (Monsivais et al., 2000; Howard and Rubel, 2010).

The effects of SR-95531 were most prominent at the later part of the train response (4-20th stimuli, bottom) than at the beginning (first-third stimuli, top) of the train (Fig. 5C). SR-95531 decreased the threshold and saturating intensities, and narrowed the dynamic range particularly in the later part of the train (Fig. $5 D-F)$; the dynamic range was $88 \pm 12 \%$ and $37 \pm 6 \%$ of controls $(n=6, p=0.0001)$ at the early and later part of the train, respectively. In addition, spike jitter was reduced in SR-95531 
A

Control

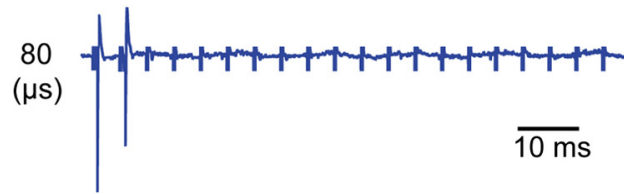

100

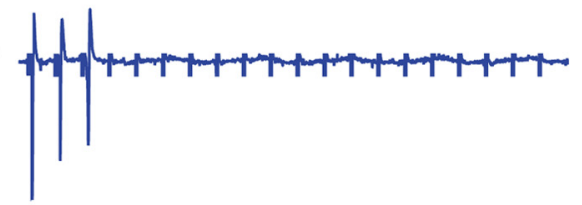

B Entire

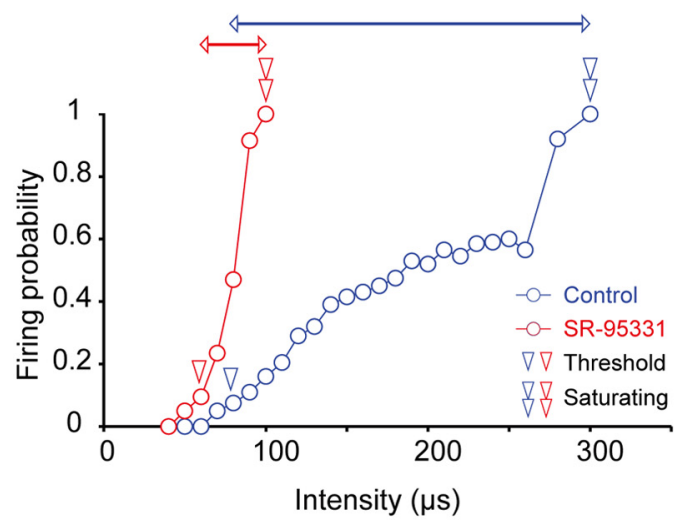

SR-95331

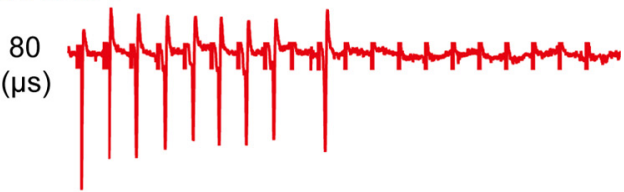

100

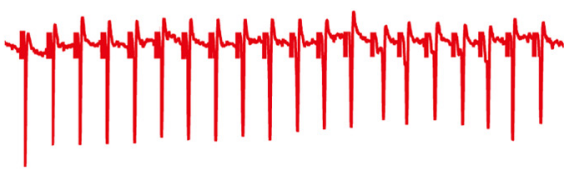

C

Early (1st-3rd)

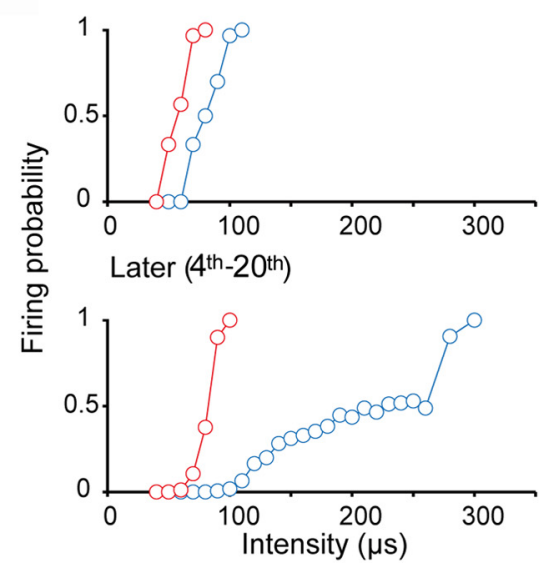

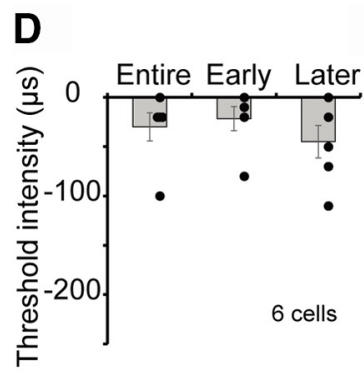

E $F$

F G
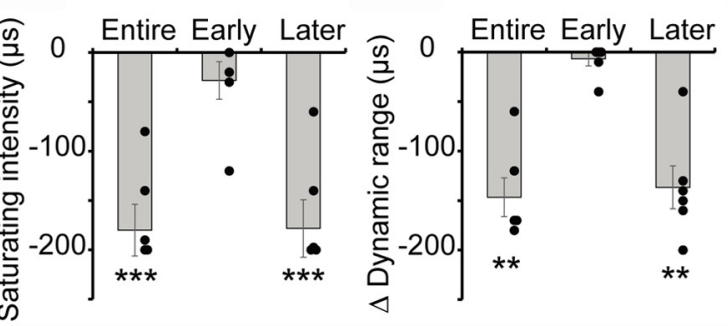

G

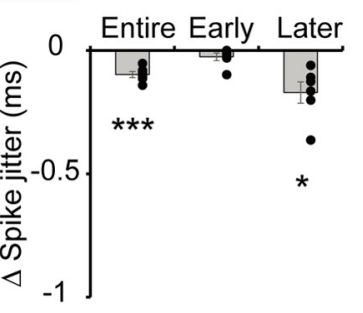

Figure 5. Polysynaptic IPSCs expanded the dynamic range of output in low-CF neurons. $A$, Spike output in response to a train of stimuli (20 pulses, $200 \mathrm{~Hz}$ ) in a low-CF neuron before (left, blue, control) and after (right, red) application of SR-95331 (100 $\mu \mathrm{M}, \mathrm{GABA}_{\mathrm{A}}$ receptor blocker). SR-95331 increased the number of spikes at each intensity. $\boldsymbol{B}$, Firing probability in $\boldsymbol{A}$ was plotted against intensity. SR-95331 decreased threshold (single arrowheads) and saturating (double arrowheads) intensities, thereby narrowing dynamic range (horizontal two-way arrows). $C$, Firing probability was plotted against intensity separately for early (first-third, top) and later (4-20th, bottom) parts. $\boldsymbol{D}$ - $\mathbf{G}$, Differences between control and SR-95331 for threshold intensity (D), saturating intensity $(\boldsymbol{E})$, dynamic range $(\boldsymbol{F})$, and spike jitter $(\boldsymbol{G})$. Differences were calculated for the entire train, for early in the train, and for later in the train. The effects of SR-95331 were predominant at the later part, reflecting the summating natures of polysynaptic IPSCS. The result also indicated that contributions of ambient GABA to the spike suppression would be small. ${ }^{*} p<0.05,{ }^{* *} p<0.01$, ${ }^{* * *} p<0.001$ between control and SR-95331.

more in the later part of the train (Fig. $5 G$ ). These results were compatible with the fact that temporal summation of IPSCs progressed and produced a plateau current at the later part of the train, so that loss of this inhibition had progressively greater effects as the stimulation proceeded (Figs. 2, 3).

\section{Effects of linear excitatory-inhibitory relationship depended on the size of unitary EPSCs}

We examined the effects of linearly coupled EPSCs and IPSCs on spike generation in a computational model of low-CF NM neurons (Fig. 6). The model neuron received multiple uEPSGs, each of which randomly varied with a jitter corresponding to that of activity of ANFs in vivo, while the tonic uIPSG was set to change in size and number in parallel with the uEPSGs (Fig. 6A; see
Materials and Methods). With an increase in the number of uEPSGs ( $4 \mathrm{nS}$ ), the number of spikes increased, but the increase was less steep with IPSGs than without IPSGs (Fig. 6A, left and middle). Accordingly, IPSGs caused a rightward shift in the firing probability; the number of uEPSGs for the maximum probability (Fig. 6C, saturation, double arrowheads) increased from 2 to 8 , whereas the number just below the appearance of spikes (threshold, single arrowheads) remained at 4 , leading to a wider dynamic range from 2 to 6 with IPSGs (horizontal two-way arrows). Thus, linearly coupled IPSCs prevented saturation of spike responses and expanded the dynamic range, consistent with physiological results in low-CF neurons (Fig. 5).

We then increased the size of the uEPSG, while preserving the linear excitatory-inhibitory relationship, and examined the ef- 
A

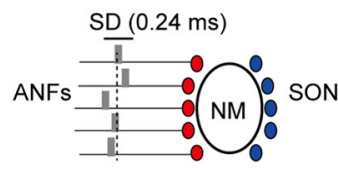

$\begin{array}{ll}\text { UEPSG (4 nS) } & \text { uEPSG (4 nS) } \\ \text { uIPSG (0 nS) } & \text { uIPSG (4 nS) }\end{array}$

uEPSG (12 nS)

uIPSG (12 nS)
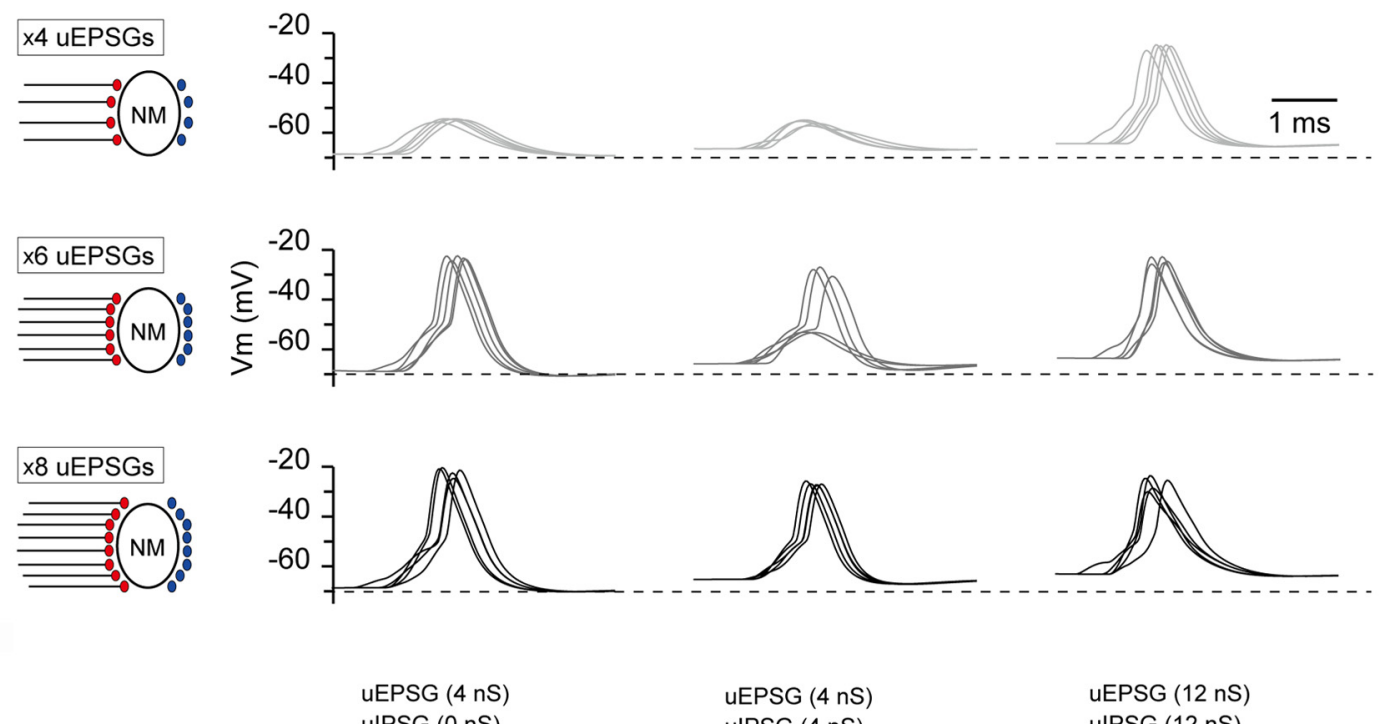

uIPSG (0 nS)
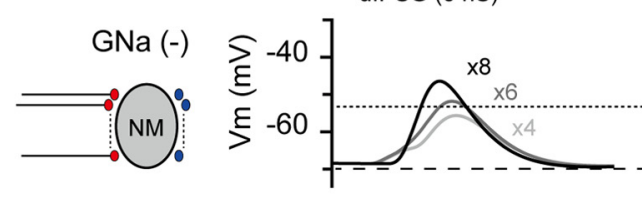

uEPSG (4 nS
uIPSG (4 nS)

UIPSG (12nS)

B

C

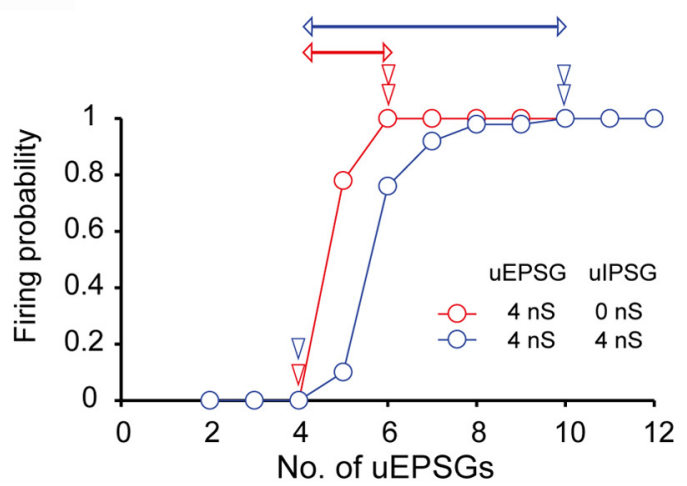

D

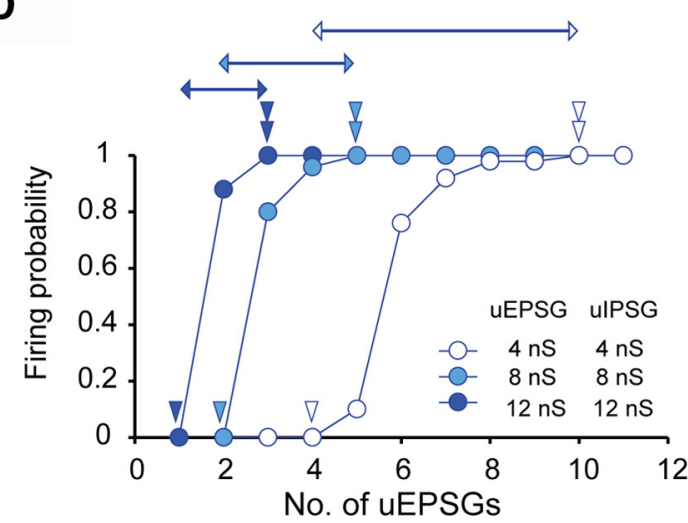

Figure 6. Linear excitatory-inhibitory relationship expanded dynamic range when uEPSCS were small. $A$, Effects of linearly coupled IPSCs on spike generation in a model of low-CF NM neurons. The model received multiple uEPSGS, and voltage responses to 4 (top), 6 (middle), and 8 (bottom) uEPSGs were shown. Five traces were superimposed. Each uEPSGs were randomly varied in the timing with a SD of $0.24 \mathrm{~ms}$. ulPSG was a tonic conductance, and the size and the number changed linearly with those of uEPSGs. Spike generation was saturated at 6 uEPSGs ( $4 \mathrm{nS}$ ) without IPSG (left), at 8 uEPSGS (4nS) with IPSG (middle), and at 4 uEPSG (12 nS) with IPSG (right). Note that resting membrane potential was depolarized particularly when IPSG was large. Broken lines indicate - 70 $\mathrm{mV} . \boldsymbol{B}$, Synaptic potentials under $\mathrm{gNa}$ of $0 \mathrm{pS} / \mu \mathrm{m}^{2}$. Three traces were superimposed for 4,6 , and 8 uEPSGs. Synaptic potential was subthreshold at 6 uEPSCS ( $4 \mathrm{nS}$ ) with IPSG (middle), whereas it exceeded spike threshold (dotted line) even at $4 \mathrm{uEPSG}$ for $12 \mathrm{nS}$ (right). C, Firing probability as a function of the number of uEPSG at $4 \mathrm{nS}$. Single arrowheads indicate the number of uEPSGs just below the appearance of spikes (threshold), and double arrowheads the number for the maximum probability (saturation). IPSG caused a rightward shift of the curve with little effects on threshold, expanding the dynamic range of responses (horizontal two-way arrows). $\boldsymbol{D}$, Firing probability was plotted against the number of uEPSG for three different uEPSG. The size and the number of ulPSGs were the same as those of uEPSGS. An increase of uEPSG caused a leftward shift of the curve, and narrowed the dynamic range of responses, indicating that the small size of uEPSG is required for the suppressive effect of linearly coupled IPSCS.

fects on the predicted input/output curve (Fig. $6 A$, right, $D$ ). The increase in uEPSG, and resulting increase in EPSP amplitude (Fig. 6B), meant that fewer inputs were needed to reach spike threshold and maximal spike probability, thus causing a prominent leftward shift of the curve and a narrowing of dynamic range. This result implies that the linearly coupled IPSCs effectively expanded the dynamic range only when UEPSG was small.
The results show that the small size of uEPSG is critical for the linear relationship between EPSC and IPSC, ensuring a wide dynamic range.

\section{Discussion}

In this study, we developed a thick-slice preparation of the chicken brainstem, and examined the relationship between 
A Low-CF Sound wave ANF Activity

NM

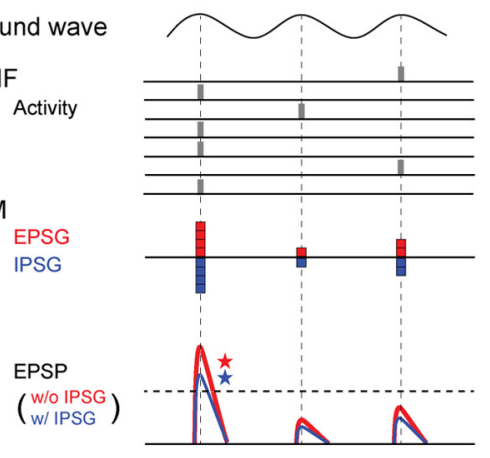

C

Middle/high-CF

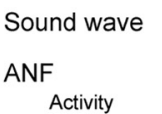

NM

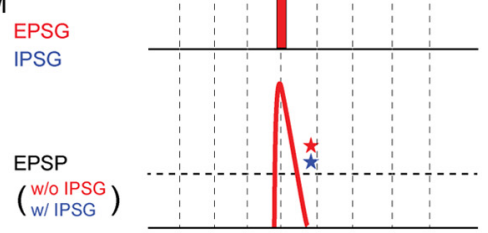

Weak sound

Spike

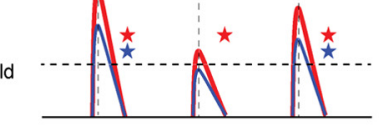

B
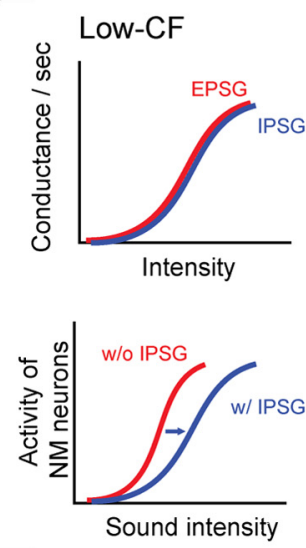

D
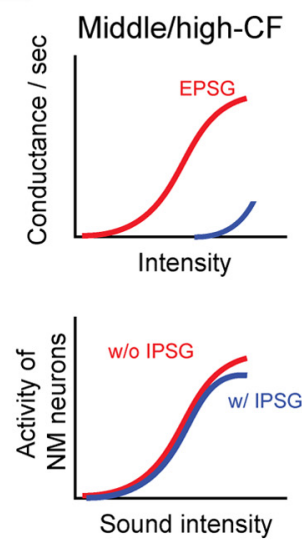

Figure 7. Roles of sound-frequency-specific excitatory-inhibitory coupling in NM. $A, C$, Schematic drawing of the input-output relationship of low-CF $(A)$ and middle/high-CF $(C)$ neurons during sound stimuli. Seven and one ANFs were assumed to innervate low-CF and middle/high-CF neurons, respectively, whereas one and two spikes were generated in each fiber for weak (left) and intense (right) sounds, respectively (top). In low-CF neurons, unitary excitatory (EPSG) and inhibitory (IPSG) conductances are small and increase in parallel with elevation of intensity (middle). Accordingly, the number of EPSPs exceeding spike threshold (broken lines and asterisks) decreases in the presence (blue) compared with the absence of the IPSG (red, bottom); the number of cycles exceeding threshold was 1, with or without IPSGs at weak sound, whereas it was 3 without the IPSG and 2 for with the IPSG, at intense sound. In middle/high-CF neurons, on the other hand, the unitary EPSG is large enough to generate spikes, whereas the IPSG is far smaller and appears at a higher intensity than the EPSG. Accordingly, the IPSG would have a smaller impact on the number of EPSPs exceeding spike threshold. Thus, spike output would reflect the number of cycles with EPSPs rather than the size of EPSPs at each cycle in middle/high-CF neurons (see Discussion); the number of cycles exceeding threshold was 1 and 2 at weak and intense sounds, respectively, with or without the IPSG. B, D, Relationships of synaptic conductance (top) and spike output of NM neurons (bottom) with sound intensity. Low-CF (B) and middle/high-CF (D) neurons. In low-CF neurons, EPSG (red) and IPSG (blue) increase in parallel (top), and hence spike output should be suppressed with IPSG (blue) compared with without IPSG (red, bottom). In middle/high-CF neurons, on the other hand, IPSG would appear at a higher intensity (blue, top), and hence effects of IPSG on spike output would be minimal (blue, bottom). Spike generation could be attenuated with IPSG at an extremely intense sound, as observed in NM neurons in vivo (Fukui et al., 2010).

excitatory and inhibitory synaptic inputs impinging on NM neurons during stimulation of ANFs. This relationship was determined differentially in a tonotopic-pathway-specific manner; IPSCs increased in parallel with EPSCs in low-CF neurons, whereas they were dominated by EPSCs in middle/ high-CF neurons. This pathway-specific coupling of excitatory and inhibitory inputs ensures a level and timing of neuronal output in the auditory circuit suited for wide intensity and frequency ranges of sound.

Tonotopic differentiation of polysynaptic inhibitory pathway IPSCs were more readily triggered in low-CF NM neurons than in middle/high-CF NM neurons, suggesting a greater importance of inhibition in control of responses to low-frequency sound. The stimulus threshold triggering EPSCs was similar across regions, excluding tonotopic differences in excitability of ANFs. Therefore, the difference in IPSC threshold must arise at NA and/or SON, through mechanisms including patterns of innervation, synaptic strength, and/or excitability of postsynaptic neurons.
In contrast to electrical stimulus thresholds, acoustic threshold for activating ANFs is high for fibers tuned to low-frequency $(<0.5 \mathrm{kHz}$; Manley et al., 1997; Fukui et al., 2006), whereas it does not differ among tonotopic regions at NA (Warchol and Dallos, 1990), suggesting that ANFs may drive spikes more efficiently in NA neurons tuned to lower frequencies. Notably, tonotopic representation changes from logarithmic to linear scales between cochlea and NA (Ryals and Rubel, 1982; Fukui and Ohmori, 2003). In addition, synaptic strength and neuronal excitability do not differ tonotopically in NA (Fukui and Ohmori, 2003; Ahn and MacLeod, 2016). Thus, one possible explanation could be that convergence of ANFs was greater at lower-CF regions in NA, as also seen in NM (Fukui and Ohmori, 2004), and this difference contributed to lower the threshold of polysynaptic IPSCs in the low-CF NM neurons.

Frequency tuning of SON neurons is broader than that of NA neurons, but the extent of frequency convergence, expected from bandwidth (Q10 dB), did not differ among tonotopic regions (Tabor et al., 2012). In addition, threshold to sound and firing 
patterns are similar between SON neurons and NA neurons (Warchol and Dallos, 1990; Tabor et al., 2012), suggesting that contributions of SON to the difference of IPSC threshold could be small. Nevertheless, a possibility remains that tonotopic differences exist at SON in the extents of convergence within a frequency band, synaptic strength, and/or neuronal excitability, which underlie the difference of IPSC threshold in NM.

\section{Intensity dependence of excitatory-inhibitory relationship}

Excitatory and inhibitory inputs increased with the intensity of stimuli in a way specific to each tonotopic region. In low-CF neurons, EPSCs and IPSCs increased with the intensity in a stepwise manner, reflecting the tight coupling of IPSCs with the activity of ANFs. In middle/high-CF neurons, on the other hand, a large EPSC appeared in an all-or-none manner, and IPSCs remained smaller than EPSCs for wide intensity ranges. These relationships were further shaped during the course of the stimulus train. EPSCs and IPSCs showed a strong depression during the train, via depletion of vesicles (Trussell, 1999). However, IPSCs were slow in decay kinetics and temporally summated to produce a plateau current at the later part, creating a time window for inhibition ( $\mathrm{Lu}$ and Trussell, 2000). Moreover, the elevation of stimulus frequency decreased the steady level of EPSCs, whereas it either did not affect or increased the level of IPSCs, implying that the relative conductance of IPSCs becomes larger at higher stimulus frequency. Because firing rate of ANFs is correlated with sound intensity, the results indicated that contributions of IPSCs to the output of NM neurons increase during intense sound. It will be of interest to examine how presynaptic inhibition of these currents differentially controls excitation vs inhibition (Brenowitz et al., 1998; Lu and Rubel, 2005; Lu, 2007).

\section{Roles of polysynaptic inhibitory inputs in NM in vivo}

Elevation of sound intensity increases the number of active ANFs and the firing rate in each fiber. Given that multiple ANFs project onto low-CF NM neurons and phase-locked spikes occur stochastically among cycles in individual ANFs, elevation of sound intensity should increase the size of EPSPs rather evenly at each cycle for low-frequency sound (Fig. $7 A, B$ ). In this situation, EPSPs may already reach a level close to spike threshold at a majority of cycles even at auditory thresholds, and hence a slight increase in the sound intensity may easily cause saturation of spike responses and narrowing the dynamic range without inhibition. Thus, the tight coupling between activation of EPSCs and IPSCs is particularly suited for the low-CF NM neurons that receive multiple converging inputs for spike generation, ensuring a wide dynamic range for spike responses (Fukui et al., 2006).

For high-frequency sound, many sound cycles fail to trigger spikes in ANFs due to the short period of the sound, although the number of these failed cycles decreases at higher sound intensity (Fig. 7C,D). Because middle/high-CF neurons receive only one or a few ANFs, and individual EPSPs are large enough to generate a spike, it is expected that elevation of sound intensity may increase the number of cycles with EPSPs rather than the size of EPSPs at each cycle. This together with the high threshold of IPSCs would contribute to the suppression of spike generation as well as the reduction of spike jitter during intense sound in neurons in vivo (Fukui et al., 2010). Although GABAergic inhibition increased spike jitter in the present study, this is expected given that EPSPs were almost perfectly synchronized and a decrease of their amplitude causes a variation of spike timing during electrical stimulation; in vivo, inhibition decreases spike jitter by preventing poorly timed EPSPs from generating spikes (Howard and Rubel,
2010). Thus, the polysynaptic IPSCs improve the phase locking, but may not expand the dynamic range in the middle/high-CF neurons.

\section{Methodological consideration}

One technical concern we had was related to the identity of the fibers activated by the electrical stimuli. In particular, the electrical stimuli might activate a broad frequency range of ANFs, which could lead to an overestimate of the polysynaptic IPSCs. However, it is not likely that these stimulus situations caused the tonotopic differences in the polysynaptic IPSCs and hence the excitatory-inhibitory relationships, because tonotopic differences are not apparent in the frequency convergence of the polysynaptic inhibitory pathways (Q10 dB; Warchol and Dallos, 1990; Tabor et al., 2012) or in the efficiency of spike induction in ANFs (threshold intensity of EPSCs; Fig. 1G). Another concern was that stimulus intensity was adjusted by changing the duration rather than the amplitude of pulses. This could cause a variation in the timing of spike induction in ANFs, which might affect the efficiency of activating the polysynaptic pathway and the excitatory-inhibitory relationship. However, this is not likely because the variation in the timing of spike induction was $<0.2 \mathrm{~ms}$ as the duration was changed (see Materials and Methods), far smaller than the time course of EPSPs in neurons within the pathway. For example, the half-amplitude widths of EPSPs were $\sim 10-20 \mathrm{~ms}$ in NA neurons (MacLeod and Carr, 2005) and 50-60 ms in SON neurons (Yang et al., 1999), which would minimize the effects of this variation on the EPSP summation and hence the spike induction in the neurons.

\section{Comparison to mammals}

The linear excitatory-inhibitory relationship in low-CF NM neurons may play a critical role in the calculation of interaural time differences at NL; it adjusts the level and timing of output of NM neurons in a sound-intensity-dependent manner, ensures appropriate sizes of EPSPs in NL neurons, and improves the binaural calculation across intensities (Nishino et al., 2008). Spherical bushy cells in AVCN, a mammalian homolog of NM neurons, also receives sound-intensity-dependent inhibitory inputs presumably via interneurons within the nucleus, which ensures precise temporal coding across intensities (Keine and Rübsamen, 2015). Interestingly, the density of inhibitory terminals differs tonotopically in AVCN, being higher at low-CF regions, as in NM (Moore and Moore, 1987; Kolston et al., 1992). In addition, excitatory inputs to spherical bushy cells are differentiated tonotopically in their size and number (Joris et al., 1994), similar to NM, suggesting that the excitatory and inhibitory balance is determined in a tonotopic-pathway-specific manner in both mammals and birds. Thus, the pathway-specific coupling of excitatory and inhibitory inputs may shape the optimum level and timing of neuronal output, thereby playing a fundamental role in the timing coding pathway in the auditory system.

\section{References}

Ahn J, MacLeod KM (2016) Target-specific regulation of presynaptic release properties at auditory nerve terminals in the avian cochlear nucleus. J Neurophysiol 115:1679-1690.

Akter N, Adachi R, Kato A, Fukaya R, Kuba H (2018) Auditory input shapes tonotopic differentiation of Kv1.1 expression in avian cochlear nucleus during late development. J Neurosci 38:2967-2980.

Brenowitz S, Trussell LO (2001) Maturation of synaptic transmission at end-bulb synapses of the cochlear nucleus. J Neurosci 21:1857-1867.

Brenowitz S, David J, Trussell L (1998) Enhancement of synaptic efficacy by presynaptic $\mathrm{GABA}_{\mathrm{B}}$ receptors. Neuron 20:135-141. 
Burger RM, Cramer KS, Pfeiffer JD, Rubel EW (2005) Avian superior olivary nucleus provides divergent inhibitory input to parallel auditory pathways. J Comp Neurol 481:6-18.

Carr CE, Boudreau RE (1991) Central projection of auditory nerve fibers in the barn owl. J Comp Neurol 314:306-318.

Code RA, Burd GD, Rubel EW (1989) Development of GABA immunoreactivity in brainstem auditory nuclei of the chick: ontogeny of gradients in terminal staining. J Comp Neurol 284:504-518.

Fukui I, Ohmori H (2003) Developmental changes in membrane excitability and morphology of neurons in the nucleus angularis of the chicken. J Physiol 548:219-232.

Fukui I, Ohmori H (2004) Tonotopic gradients of membrane and synaptic properties for neurons of the chicken nucleus magnocellularis. J Neurosci 24:7514-7523.

Fukui I, Sato T, Ohmori H (2006) Improvement of phase information at low sound frequency in nucleus magnocellularis of the chicken. J Neurophysiol 96:633-641.

Fukui I, Burger RM, Ohmori H, Rubel EW (2010) GABAergic inhibition sharpens the frequency tuning and enhances phase locking in chicken nucleus magnocellularis neurons. J Neurosci 30:12075-12083.

Hackett JT, Jackson H, Rubel EW (1982) Synaptic excitation of the second and third order auditory neurons in the avian brain stem. Neuroscience 7:1455-1469.

Hines ML, Morse T, Migliore M, Carnevale NT, Shepherd GM (2004) Model DB: a database to support computational neuroscience. J Comput Neurosci 17:7-11.

Howard MA, Rubel EW (2010) Dynamic spike thresholds during synaptic integration preserve and enhance temporal response properties in the avian cochlear nucleus. J Neurosci 30:12063-12074.

Hyson RL, Reyes AD, Rubel EW (1995) A depolarizing inhibitory response to GABA in brainstem auditory neurons of the chick. Brain Res 677: $117-126$.

Jhaveri S, Morest DK (1982) Neuronal architecture in nucleus magnocellularis of the chicken auditory system with observations on nucleus laminaris: a light and electron microscope study. Neuroscience 7:809-836.

Joris PX, Smith PH, Yin TC (1994) Enhancement of neural synchronization in the anteroventral cochlear nucleus: II. Responses in the tuning curve tail. J Neurophysiol 71:1037-1051.

Keine C, Rübsamen R (2015) Inhibition shapes acoustic responsiveness in spherical bushy cells. J Neurosci 35:8579-8592.

Kolston J, Osen KK, Hackney CM, Ottersen OP, Storm-Mathisen J (1992) An atlas of glycine- and GABA-like immunoreactivity and colocalization in the cochlear nuclear complex of the guinea pig. Anat Embryol 186: 443-465.

Köppl C (1994) Auditory nerve terminals in the cochlear nucleus magnocellularis: differences between low and high frequencies. J Comp Neurol 339:438-446.

Köppl C (1997) Phase locking to high frequencies in the auditory nerve and cochlear nucleus magnocellularis of the barn owl, Tyto alba. J Neurosci 17:3312-3321.

Kuba H, Ohmori H (2009) Roles of axonal sodium channels in precise auditory time coding at nucleus magnocellularis of the chick. J Physiol $587: 87-100$

Kuo SP, Bradley LA, Trussell LO (2009) Heterogeneous kinetics and pharmacology of synaptic inhibition in the chick auditory brainstem. J Neurosci 29:9625-9634.

Lachica EA, Rübsamen R, Rubel EW (1994) GABAergic terminals in nucleus maznocellularis and laminaris originate from the superior olivary nucleus. J Comp Neurol 348:403-418.

Lu T, Trussell LO (2000) Inhibitory transmission mediated by asynchronous transmitter release. Neuron 26:683-694.

Lu T, Trussell LO (2001) Mixed excitatory and inhibitory GABA-mediated transmission in chick cochlear nucleus. J Physiol 535:125-131.

Lu Y (2007) Endogenous mGluR activity suppresses GABAergic transmission in avian cochlear nucleus magnocellularis neurons. J Neurophysiol 97:1018-1029.

Lu Y, Rubel EW (2005) $\mathrm{GABA}_{\mathrm{B}}$ receptor activation modulates $\mathrm{GABA}_{\mathrm{A}}$ receptor-mediated inhibition in chicken nucleus magnocellularis neurons. J Neurophysiol 93:1418-1428.

MacLeod KM, Carr CE (2005) Synaptic physiology in the cochlear nucleus angularis of the chick. J Neurophysiol 93:2520-2529.

Manley GA, Köppl C, Yates GK (1997) Activity of primary auditory neurons in the cochlear ganglion of the emu Dromaius novaehollandiae: spontaneous discharge, frequency tuning, and phase locking. J Acoust Soc Am 101:1560-1573.

Monsivais P, Rubel EW (2001) Accommodation enhances depolarizing inhibition in central neurons. J Neurosci 21:7823-7830.

Monsivais P, Yang L, Rubel EW (2000) GABAergic inhibition in nucleus magnocellularis: implications for phase locking in the avian auditory brainstem. J Neurosci 20:2954-2963.

Moore JK, Moore RY (1987) Glutamic acid decarboxylase-like immunoreactivity in brainstem auditory nuclei of the rat. J Comp Neurol 260: 157-174.

Nishino E, Yamada R, Kuba H, Hioki H, Furuta T, Kaneko T, Ohmori H (2008) Sound-intensity-dependent compensation for the small interaural time difference cue for sound source localization. J Neurosci 28:7153-7164.

Oline SN, Burger RM (2014) Short-term synaptic depression is topographically distributed in the cochlear nucleus of the chicken. J Neurosci 34:1314-1324.

Parks TN, Rubel EW (1978) Organization and development of the brain stem auditory nuclei of the chicken: primary afferent projections. J Comp Neurol 180:439-448.

Rathouz M, Trussell L (1998) Characterization of outward currents in neurons of the avian nucleus magnocellularis. J Neurophysiol 80:2824-2835.

Roberts MT, Seeman SC, Golding NL (2014) The relative contributions of MNTB and LNTB neurons to inhibition in the medial superior olive assessed through single and paired recordings. Front Neural Circuits 8:49.

Rothman JS, Manis PB (2003) The roles potassium currents play in regulating the electrical activity of ventral cochlear nucleus neurons. J Neurophysiol 89:3097-3113.

Rubel EW, Parks TN (1975) Organization and development of brain stem auditory nuclei of the chicken: tonotopic organization of N. magnocellularis and N. laminaris. J Comp Neurol 164:411-433.

Ryals BM, Rubel EW (1982) Patterns of hair cell loss in chick basilar papilla after intense auditory stimulation 1: frequency organization. Acta Otolaryngol 93:205-210.

Saunders JC, Coles RB, Gates GR (1973) The development of auditory evoked responses in the cochlea and cochlear nuclei of the chick. Brain Res 63:59-74.

Sullivan WE, Konishi M (1984) Segregation of stimulus phase and intensity coding in the cochlear nucleus of the barn owl. J Neurosci 4:1787-1799.

Tabor KM, Coleman WL, Rubel EW, Burger RM (2012) Tonotopic organization of the superior olivary nucleus in the chicken auditory brainstem. J Comp Neurol 520:1493-1508.

Trussell LO (1999) Synaptic mechanisms for coding timing in auditory neurons. Annu Rev Physiol 61:477-496.

von Bartheld CS, Code RA, Rubel EW (1989) GABAergic neurons in brainstem auditory nuclei of the chick: distribution, morphology, and connectivity. J Comp Neurol 287:470-483.

Warchol ME, Dallos P (1990) Neural coding in the chick cochlear nucleus. J Comp Physiol A 166:721-734.

Yamada R, Okuda H, Kuba H, Nishino E, Ishii TM, Ohmori H (2013) The cooperation of sustained and phasic inhibitions increases the contrast of ITD-tuning in low-frequency neurons of the chick nucleus laminaris. J Neurosci 33:3927-3938.

Yang L, Monsivais P, Rubel EW (1999) The superior olivary nucleus and its influence on nucleus laminaris: a source of inhibitory feedback for coincidence detection in the avian auditory brainstem. J Neurosci 19: 2313-2325.

Young SR, Rubel EW (1983) Frequency-specific projections of individual neurons in chick brainstem auditory nuclei. J Neurosci 3:1373-1378.

Zhang S, Trussell LO (1994) A characterization of EPSPs in the avian nucleus magnocellularis. J Neurophysiol 72:705-718. 